\title{
FLAVONOIDES E SESQUITERPENOS DE Croton pedicellatus Kunth.\#
}

Elton Luz Lopes, Manoel Andrade Neto, Edilberto Rocha Silveira e Otilia Deusdênia Loiola Pessoa*

Departamento de Química Orgânica e Inorgânica, Universidade Federal do Ceará, CP 12200, 60971-270 Fortaleza - CE, Brasil Raimundo Braz-Filho ${ }^{*}$

Departamento de Química, Universidade Estadual do Norte Fluminense, 28013-602 Campos dos Goytacazes - RJ, Brasil

Recebido em 25/4/12; aceito em 7/8/12; publicado na web em 5/10/12

FLAVONOIDS AND SESQUITERPENES OF Croton pedicellatus Kunth. The chemical investigation of the ethanolic extract from leaves of Croton pedicellatus yielded the bis-nor-sesquiterpenes blumenol A and blumenol A glucoside, along with the flavonoids: tiliroside, 6"-O-p-coumaroyl- $\beta$-galactopyranosyl- kaempferol, 6"-O-p-coumaroyl- $\beta$-glucopyranosyl-3"-methoxy- kaempferol, kaempferol, 3-glucopyranosyl-quercetin and alpinumisoflavone, as well as 4-hydroxy-3,5-dimethoxybenzoic acid. The identification of all isolated compounds was performed by spectrometric methods, including HR-ESI-MS, 1D and 2D NMR experiments, and by comparison with previously-described physical and spectral data.

Keywords: Croton pedicellatus; blumenol A; tiliroside.

\section{INTRODUÇÃO}

A subfamília Crotonoideae, pertencente à família Euphorbiaceae, consiste de aproximadamente 2.400 espécies, agrupadas em 67 gêneros e 12 tribos. ${ }^{1} \mathrm{O}$ gênero Croton, o segundo maior e mais diverso desta família, é constituído por cerca de 1.200 espécies, com distribuição pantropical, porém, com significativa concentração nas Américas e África. ${ }^{2}$

Muitas espécies de Croton se desenvolvem em locais como margens de estradas, rios e clareiras de matas. Essas e outras características ecológicas, como a produção massiva de flores e frutos durante a maior parte do ano, fazem dos membros deste gênero candidatos ideais para a restauração de florestas degradadas. Neste contexto, pode-se citar o exemplo de Croton urucurana Spreng., o qual foi cultivado com a finalidade de reflorestamento de matas ciliares no Sul do Brasil e em outras regiões. ${ }^{3}$

Várias espécies de Croton são reconhecidas como medicinais, particularmente nas Américas, África e Ásia. ${ }^{4}$ Seus benefícios terapêuticos abrangem tanto doenças complexas como câncer, diabetes e malária, bem como constipação intestinal, diarreia, cicatrização de feridas externas, febre, inflamações, dores e úlceras. ${ }^{5} \mathrm{O}$ grande leque de atividades descritas para plantas deste gênero é reflexo da alta diversidade química produzida por esse grupo, particularmente rico em metabólitos secundários, especialmente terpenoides, alcaloides e flavonoides. ${ }^{6}$ Os terpenoides são os metabólitos secundários predominantes no gênero Croton, com destaque para os diterpenos de esqueleto clerodano, que formam um grupo de substâncias extremamente diversificadas, contando com mais de 800 compostos já conhecidos. ${ }^{5}$ Embora em proporções menores, outros diterpenos, como cembranoide, halimano, pimarano, caurano, labdano e traquilobano, também foram encontrados. ${ }^{7}$ Triterpenos pentacíclicos e esteroides também são frequentemente relados. ${ }^{8}$ As plantas deste gênero também são produtoras de diferentes classes de alcaloides, fato que aumenta consideravelmente a importância do gênero sob o ponto de vista farmacológico. Compostos fenólicos têm sido frequentemente relatados, predominando os flavonoides, lignoides e proantocianidinas. Óleos

*e-mail: opessoa@ufc.br

\#Artigo em homenagem ao Prof. Otto R. Gottlieb (31/8/1920-19/6/2011)

\&Professor visitante emérito - FAPERJ/UENF/UFRRJ voláteis, ricos em mono e sesquiterpenos, e algumas vezes compostos derivados do chiquimato são outra característica do gênero. ${ }^{4}$

Apesar do grande número de substâncias já isoladas e das atividades biológicas investigadas, ainda é muito pequeno o número de espécies de Croton estudadas, quer seja do ponto de vista químico ou farmacológico. Desta forma, a prospecção química aliada à farmacológica envolvendo plantas deste gênero é de extrema importância para um melhor conhecimento do potencial dessas plantas. Neste trabalho apresenta-se o resultado obtido com o estudo químico de Croton pedicellatus, o qual está sendo relatado pela primeira vez.

\section{PARTE EXPERIMENTAL}

\section{Procedimentos experimentais gerais}

Os espectros no infravermelho foram registrados em espectrômetro da Perkin-Elmer, Spectrum 100 FTIR equipado com assessório UATR. Os espectros de ressonância magnética nuclear (1D e 2D) foram obtidos em espectrômetro, modelo Avance DRX-500 (Bruker) equipado com sonda de $5 \mathrm{~mm}$, operando nas frequências de 500 e 125 MHz para nuclídeos hidrogênio-1 e carbono-13, respectivamente. Os espectros de massa de alta resolução foram obtidos em espectrômetro LCMS-IT-TOF (Shimadzu), equipado com fonte de ionização por eletrospray. As amostras foram analisadas por injeção direta $(5 \mu \mathrm{L})$ utilizando como fase móvel a mistura $\mathrm{MeOH}: \mathrm{H}_{2} \mathrm{O}$ 9:1. A separação dos compostos foi realizada em cromatógrafo líquido de alta eficiência da Shimadzu-UFLC com detector UV-Vis com arranjo de diodos modelo FTD-M20A empregando coluna semipreparativa Phenomenex (C-18, $250 \times 10 \mathrm{~mm}$ ), com partículas de $5 \mu \mathrm{m}$. Nas cromatografias de adsorção em coluna (CC) utilizou-se gel de sílica 60 da Vetec (70-230 mesh) e Merck (40-63mesh), enquanto as cromatografias em camada delgada (CCD) foram realizadas em cromatofolha de alumínio (Merck) com indicador de fluorescência $\left(\mathrm{F}_{254}\right)$ e reveladas por aspersão de solução de vanilina/ácido perclórico/EtOH, seguida de aquecimento em estufa $\left(\approx 100{ }^{\circ} \mathrm{C}\right)$.

\section{Material vegetal}

As folhas de $C$. pedicellatus foram coletadas no município de Natal - Rio Grande do Norte, em agosto de 2008 (06 05'19,7” S 
e $\left.35^{\circ} 07^{\prime} 33,0^{\prime \prime} \mathrm{W}\right)$. A autenticação do material vegetal foi realizada pelo Prof. E. de P. Nunes (Departamento de Biologia - UFC). Uma exsicata representando a coleta da planta encontra-se depositada no Herbário Prisco Bezerra (EAC) da Universidade Federal do Ceará, sob o número 44459.

\section{Extração e isolamento}

As folhas (1 kg), após secas em estufa com ventilação e trituradas, foram submetidas à extração com $10 \mathrm{~L}$ de etanol $(3 \mathrm{x})$, gerando $80 \mathrm{~g}$ de extrato bruto após destilação do solvente em evaporador rotativo sob pressão reduzida a $40{ }^{\circ} \mathrm{C}$. Uma alíquota de $6 \mathrm{~g}$ do extrato foi fracionada sobre 56,5 $\mathrm{g}$ de gel de sílica $60(63-200 \mu \mathrm{m})$ utilizando como fase móvel os eluentes hexano, $\mathrm{CH}_{2} \mathrm{Cl}_{2}$, AcOEt e $\mathrm{MeOH}$, puros ou em misturas binárias na proporção de 1:1, seguindo ordem crescente de polaridade. A fração obtida com AcOEt apresentou um precipitado amarelo que, depois de separado por filtração $(410 \mathrm{mg})$, foi submetido à cromatografia flash $(40-63 \mu \mathrm{m})$, empregando como eluente AcOEt/MeOH (97:3), resultando no isolamento de um sólido amarelo caracterizado como canferol $(\mathbf{1}, 8,6 \mathrm{mg}){ }^{9}$ Uma outra alíquota do extrato bruto $(40 \mathrm{~g})$ foi dissolvida em $200 \mathrm{~mL}$ da mistura metanol/ água $(1: 1)$ e submetida à partição líquido-líquido $(4$ x $50 \mathrm{~mL})$ com hexano, $\mathrm{CH}_{2} \mathrm{Cl}_{2}$, AcOEt e n-BuOH $(2 \times 30 \mathrm{~mL})$, resultando nas frações CPFE-H, CPFE-D, CPFE-Ac e CPFE-Bu, respectivamente. A fração CPFE-D $(6,52 \mathrm{~g})$ foi submetida à CC sobre gel de sílica 60 $(63-200 \mu \mathrm{m})$ resultando em 7 novas frações (CPFE-D 1 a 7). CPFE-D 2 (476 mg) foi submetida à cromatografia flash $(43-60 \mu \mathrm{m})$ utilizando como fase móvel (hexano/AcOEt 7:3) dando origem a 70 frações que, após analisadas por CCD, foram reunidas em 6 frações (F1-F6). F2 (26,8 mg) foi ressubmetida à cromatografia flash utilizando hexano/ AcOEt $(8,5: 1,5)$, gerando cristais aciculares, e foi identificada como alpinumisoflavona $(2,15,8 \mathrm{mg}) .{ }^{10} \mathrm{~A}$ fração CPFE-Ac $(6,8 \mathrm{~g})$ foi submetida à CC sobre gel de sílica desativada (sílica imersa em EtOH por $3 \mathrm{~h}$, seguida por filtração a vácuo e lavagem com AcOEt) e como eluentes foram utilizados hexano e AcOEt puros ou em mistura binária seguindo ordem crescente de polaridade, seguido de $\mathrm{MeOH}$, gerando 8 frações (CPFE-Ac 1 a 8), após monitoramento em CCD. A fração CPFE-Ac3 $\left(60 \mathrm{mg}\right.$ ) foi submetida à CLAE (fase móvel $\mathrm{MeOH} / \mathrm{H}_{2} \mathrm{O} 5,5: 4,5$; fluxo $4,72 \mathrm{~mL} / 8 \mathrm{~min}$ ) resultando no isolamento de dois compostos sólidos, um incolor e outro amarelo, caracterizados como blumenol A (3, $\mathrm{T}_{\mathrm{R}}$ 6,2 $\left.\mathrm{min}, 6,1 \mathrm{mg}\right)^{11} \mathrm{e}$ ácido 4-hidroxi-3,5-dimetoxibenzoico (4, $\mathrm{T}_{\mathrm{R}}$ 4,6 min, 12,1 mg), ${ }^{12}$ respectivamente. Uma alíquota de CPFE-Ac 4 (400 mg) também foi fracionada por CLAE (fase móvel $\mathrm{H}_{2} \mathrm{O} / \mathrm{ACN}$ $7,3: 2,7$; fluxo $4,72 \mathrm{~mL} / 20 \mathrm{~min}$ ) resultando no isolamento dos compostos: 6"- $O$-p-cumaroil- $\beta$-galactopiranosilcanferol $\left(5, \mathrm{~T}_{\mathrm{R}} 14,9 \mathrm{~min}\right.$; $7,6 \mathrm{mg}){ }^{13} 6$ " $-O$-p-cumaroil- $\beta$-glicopiranosilcanferol (tilirosídeo) $\left(\mathbf{6}, \mathrm{T}_{\mathrm{R}} 15,6 \mathrm{~min} \text {; pf } 218,1-219,4^{\circ} \mathrm{C}, 102,3 \mathrm{mg}\right)^{13}$ e 6"-O-p-cumaroil$\beta$-glicopiranosil-3'-metoxicanferol $\left(7, \mathrm{~T}_{\mathrm{R}} 17,8 \mathrm{~min} ; 15,8 \mathrm{mg}\right) .{ }^{14} \mathrm{~A}$ fração CPFE-Ac $5(1,2 \mathrm{~g})$ após ser submetida à CC sobre gel de sílica desativada e eluída com hexano, AcOEt e $\mathrm{MeOH}$, gerou 8 subfrações denominadas de CPFE-Ac 5 (1-8). 60 mg de CPFE-Ac5 (1) foi fracionada por CLAE (fase móvel $\mathrm{H}_{2} \mathrm{O} / \mathrm{ACN} 9,3: 2,7 ; 4,72 \mathrm{~mL} / 10 \mathrm{~min}$ ) resultando no isolamento de 3-glicopiranosilquercetina $\left(8, T_{R} 4,6\right.$ min; $5,8 \mathrm{mg}) .{ }^{15}$ De forma semelhante, $120 \mathrm{mg}$ de CPFE-Ac5 (5) também foram aplicadas à CLAE (fase móvel $\mathrm{H}_{2} \mathrm{O} / \mathrm{ACN} 8,5: 1,5 ; 4,72 \mathrm{~mL} / 10 \mathrm{~min}$ ) fornecendo o composto roseosídeo $\left(9, \mathrm{~T}_{\mathrm{R}} 7,5 \mathrm{~min} ; 14,8 \mathrm{mg}\right){ }^{16}$

\section{RESULTADOS E DISCUSSÃO}

A prospecção química do extrato EtOH das folhas de C. pedicellatus resultou no isolamento de 9 metabólitos secundários, cujas estruturas foram determinadas utilizando dados de $\mathrm{RMN}{ }^{1} \mathrm{H} \mathrm{e}{ }^{13} \mathrm{C}$, incluindo experimentos bidimensionais como COSY, HSQC e HMBC

e NOESY, além de IV e EMAR. As estruturas dos metabólitos canferol (1), alpinumisoflavona (2) e 3-glicopiranosilquercetina (8) e do ácido 4-hidroxi-3,5-dimetoxibenzoico (4) (Figura 1) foram facilmente determinadas, entretanto, as estruturas dos demais flavonoides, em virtude da semelhança estrutural, assim como dos dois sesquiterpenos exigiram maior atenção quanto à interpretação dos dados espectrais.<smiles>O=c1c(O)c(-c2ccc(O)cc2)oc2cc(O)cc(O)c12</smiles><smiles>CC1=CC(=O)CC(C)(C)[C@@]1(O)/C=C/[C@H](C)O</smiles>
3: $\mathrm{R}=\mathrm{H}$ 9: $R=G / c$<smiles>CC1(C)C=Cc2c(cc3occ(-c4ccc(O)cc4)c(=O)c3c2O)O1</smiles>

2<smiles>COc1cc(C(=O)O)cc(OC)c1O</smiles><smiles></smiles><smiles>O=C(O)C(O)Oc1c(-c2ccc(O)c(O)c2)oc2cc(O)cc(O)c2c1=O</smiles>

Figura 1. Estruturas das substâncias isoladas de C. pedicellatus

O composto 3, um sólido branco com faixa de fusão de $110-112{ }^{\circ} \mathrm{C}$, apresentou a fórmula molecular $\mathrm{C}_{13} \mathrm{H}_{20} \mathrm{O}_{3}$, a qual foi deduzida por espectrometria de massa de alta resolução $(\mathrm{m} / \mathrm{z}, 223,1339$, $\left.[\mathrm{M}-\mathrm{H}]^{-}\right)$. No espectro de $\mathrm{RMN}{ }^{1} \mathrm{H}\left(500 \mathrm{MHz}, \mathrm{CDCl}_{3}\right.$ ) foram observados três sinais de hidrogênios olefínicos em $\delta 5,90(s l, \mathrm{H}-2), 5,78$ (d, $J=15,5 \mathrm{~Hz}, \mathrm{H}-7)$ e $5,84(d d, J=15,5$ e $5,5 \mathrm{~Hz}, \mathrm{H}-8)$, quatro grupos metílicos em $\delta 1,28(d, J=6,5 \mathrm{~Hz}, \mathrm{Me}-10), 1,89(s, \mathrm{Me}-11), 1,07(s$, Me-13) e 1,01 ( $s$, Me-12), dois prótons metilênicos em $\delta 2,44$ (H-6a) e 2,23 (H-6b) $(d, J=17,4 \mathrm{~Hz})$ e um hidrogênio ligado a carbono oxigenado em $\delta 4,40(q, J=6,5 \mathrm{~Hz}, \mathrm{H}-9)$. O espectro de RMN ${ }^{13} \mathrm{C}(125$ $\mathrm{MHz}, \mathrm{CDCl}_{3}$ ) exibiu 13 linhas espectrais, corroborando os dados de hidrogênio e destacando, entre outros, a presença de uma carbonila de cetona conjugada ( $\delta 198,4, \mathrm{C}-1)$ e dos carbonos oxigenados com deslocamento químico em $\delta$ 79,2 (C-4) e 68,2 (C-9) (Tabela 1). A combinação da fórmula molecular com os dados de RMN sugere a estrutura de um bis-nor-sesquiterpeno. Uma análise detalhada dos dados de RMN e comparação com informações da literatura levaram à conclusão de que o composto se tratava do sesquiterpeno blumenol A, ${ }^{17}$ previamente isolado de Croton tiglium. ${ }^{11}$

O composto 5, sólido amorfo amarelo, pf 208,6-209, $4^{\circ} \mathrm{C}$, teve sua fórmula molecular $\mathrm{C}_{30} \mathrm{H}_{26} \mathrm{O}_{13}$ determinada através do espectro de massa de alta resolução obtido com ionização por eletrospray $\left(\mathrm{m} / \mathrm{z}, 617,1243,[\mathrm{M}+\mathrm{Na}]^{+}\right)$. O espectro de absorção na região do infravermelho apresentou bandas características de deformação axial de grupos hidroxila $\left(3400 \mathrm{~cm}^{-1}\right)$ e carbonila $\left(1690 \mathrm{~cm}^{-1}\right)$, além 
Tabela 1. Dados de $\mathrm{RMN}{ }^{1} \mathrm{H}(500 \mathrm{MHz})$ e ${ }^{13} \mathrm{C}(125 \mathrm{MHz})$ de $3\left(\mathrm{CDCl}_{3}\right)$ e $9(\mathrm{MeOD})^{\mathrm{a}}$

\begin{tabular}{|c|c|c|c|c|}
\hline & $3 \delta_{\mathrm{C}}$ & $3 \delta_{\mathrm{H}}$ & $9 \delta_{\mathrm{C}}$ & $9 \delta_{\mathrm{H}}$ \\
\hline 1 & 198,45 & - & 201,35 & - \\
\hline 2 & 127,03 & $5,90(s l)$ & 127,33 & $5,87(m)$ \\
\hline 3 & 163.36 & - & 167,39 & - \\
\hline 4 & 79,24 & - & 80,15 & - \\
\hline 5 & 41,38 & - & 42,57 & - \\
\hline \multirow[t]{2}{*}{6} & 49,90 & $2,44(d, 17,4)$ & 50,85 & $2,52(d, 16,9)$ \\
\hline & & $2,23(d, 17,4)$ & & $2,15(d, 16,9)$ \\
\hline 7 & 129,23 & $5,78(d, 15,5)$ & 131,69 & $5,86(m)$ \\
\hline 8 & 135,94 & $5,84(d d, 15.5 ; 5.5)$ & 135,43 & $5,86(m)$ \\
\hline 9 & 68,22 & $4,40(q, 6,5)$ & 77,43 & 4,42 (quint, 5,4) \\
\hline 10 & 23,92 & $1,28(d, 6,5)$ & 21,33 & $1,29(d, 6,3)$ \\
\hline 11 & 19,14 & $1,89(s)$ & 19,70 & $1,92(s)$ \\
\hline 12 & 24,23 & $1,01(s)$ & 24,83 & $1,03(s)$ \\
\hline 13 & 23,11 & $1,07(s)$ & 23,57 & $1,04(s)$ \\
\hline $1^{\prime}$ & & & 102,89 & $4,34(d, 7,8)$ \\
\hline $2^{\prime}$ & & & 75,39 & $3,17(t, 7,8)$ \\
\hline $3^{\prime}$ & & & 78,25 & $3,25(m)$ \\
\hline $4^{\prime}$ & & & 71,80 & $3,24(m)$ \\
\hline 5 & & & 78,17 & $3,34(m)$ \\
\hline \multirow[t]{2}{*}{$6^{\prime}$} & & & 62,98 & $3,85(d d, 11,7$ e 1,4$)$ \\
\hline & & & & $3,63(d d, 11,7$ e 5,2$)$ \\
\hline
\end{tabular}

${ }^{a}$ Constantes de acoplamento $(J)$ em Hz e deslocamentos químicos $(\delta)$ em ppm.

de bandas esqueletais $\left(1610\right.$ e $\left.1540 \mathrm{~cm}^{-1}\right)$. O espectro de RMN ${ }^{1} \mathrm{H}$ $\left(500 \mathrm{MHz}, \mathrm{CDCl}_{3}\right)$ apresentou sinais em $\delta 8,48(2 \mathrm{H}, d, J=8,6 \mathrm{~Hz}$, H-2'/6') e 7,19 (2H, $d, J=8,6 \mathrm{~Hz}, \mathrm{H}-3$ '/5') para hidrogênios em posição orto em um sistema aromático $p$-substituído e sinais em $\delta$ $6,73(1 \mathrm{H}, d, J=1,9 \mathrm{~Hz}, \mathrm{H}-8)$ e $6,71(1 \mathrm{H}, d, J=1,9 \mathrm{~Hz}, \mathrm{H}-6)$ para hidrogênios meta posicionados, conforme valores das constantes de acoplamentos. Estes dados foram compatíveis com uma estrutura flavonoídica substituída nas posições 5,7 e 4' ${ }^{10}$ Os sinais em $\delta 7,84$ ( $1 \mathrm{H}, d, J=15,8 \mathrm{~Hz}, \mathrm{H}-7$ '”) e $6,48(1 \mathrm{H}, d, J=15,8 \mathrm{~Hz}, \mathrm{H}-8$ "') revelaram uma dupla ligação com configuração trans, que em conjunto com os sinais em $\delta 7,52(1 \mathrm{H}, d, J=8,9 \mathrm{~Hz}, \mathrm{H}-2$ "', $/ 6 " ')$ e 7,16 $(1 \mathrm{H}$, $d, J=8,9 \mathrm{~Hz}, \mathrm{H}-3$ " " $/ 5$ "'), indicaram uma unidade $p$-cumaroil. ${ }^{11} \mathrm{Os}$ sinais em $\delta 6,12(1 \mathrm{H}, d, J=7,7 \mathrm{~Hz}, \mathrm{H}-1$ "), 4,94 (1H, $d d, J=11,0$ e 7,6 Hz, H-6"a), 4,80 (1H, $t, J=9,2 \mathrm{~Hz}, \mathrm{H}-2$ "), 4,82 (1H, $d d, J=$ 11,0 e 4,5 Hz, H-6"b), 4,43 (1H, $m$, H-4") e 4,32 (2H, $m$, H-3"/5") mostraram a presença de uma unidade de açúcar. A combinação dos espectros de RMN ${ }^{13} \mathrm{C}$-PND e DEPT 135 indicou a presença de grupos carbonilas, anéis aromáticos e de uma unidade monossacarídica, corroborando com as informações obtidas do espectro de RMN ${ }^{1} \mathrm{H}$. Os dados espectrais observados quando comparados àqueles disponíveis na literatura foram compatíveis com a galactose. Deste modo, a estrutura do composto $\mathbf{5}$ foi identificada como sendo o flavonoide 6 "-O-p-cumaroil- $\beta$-galactopiranosilcanferol. ${ }^{9}$

O composto 6, sólido amorfo amarelo, pf $218,1-219,4{ }^{\circ} \mathrm{C}$, também teve sua fórmula molecular $\mathrm{C}_{30} \mathrm{H}_{26} \mathrm{O}_{13}$ determinada através do espectro de massa de alta resolução $\left(\mathrm{m} / \mathrm{z}, 617,1223,[\mathrm{M}+\mathrm{Na}]^{+}\right)$. O espectro de absorção na região do infravermelho apresentou bandas características de deformação axial de grupos hidroxila $\left(3461 \mathrm{~cm}^{-1}\right)$, carbonila $\left(1710 \mathrm{~cm}^{-1}\right)$ e bandas esqueletais $\left(1606 \mathrm{e} 1500 \mathrm{~cm}^{-1}\right)$. Os espectros de $\mathrm{RMN}{ }^{1} \mathrm{H}$ (500 MHz, MeOD) e ${ }^{13} \mathrm{C}(125 \mathrm{MHz})$ revelaram uma estreita semelhança com aqueles do composto $\mathbf{6}$. As principais diferenças foram quanto aos dados referentes à unidade de açúcar que, após análise meticulosa e comparação com dados da literatura, foram compatíveis com uma unidade de glicose, cuja única diferença da galactose é quanto à posição da hidroxila em C-4". Estes dados permitiram determinar a estrutura do composto 6 como 6"-O- $p$ cumaroil- $\beta$-glicopiranosilcanferol, conhecido como tilirosídeo ${ }^{13} \mathrm{e}$ frequente no gênero Croton. ${ }^{18}$

O composto 7, obtido como um sólido amarelo, pf 203,2-204,7 ${ }^{\circ} \mathrm{C}$ e fórmula molecular $\mathrm{C}_{31} \mathrm{H}_{28} \mathrm{O}_{14}\left(\mathrm{~m} / z, 647,1330,[\mathrm{M}+\mathrm{Na}]^{+}\right)$também mostrou, através de seus dados espectrais, ter uma estrutura semelhante a 5. Os espectros de $\mathrm{RMN}{ }^{1} \mathrm{H} \mathrm{e}{ }^{13} \mathrm{C}$ evidenciaram a presença de um grupo metoxila, em $\delta 3,90$ e 56,8, respectivamente, cuja posição foi definida através do espectro HMBC, bem como diferença no padrão de substituição do anel B quando comparado ao de 5. Para este anel foram observados sinais em $\delta 7,85(d, J=1,9 \mathrm{~Hz}, \mathrm{H}-2$ '), 7,54 ( $d d$, $J=1,9$ e $8,4 \mathrm{~Hz}, \mathrm{H}-6$ ') e $6,82\left(d, J=8,4 \mathrm{~Hz}, \mathrm{H}-5^{\prime}\right)$, indicando um sistema de spins do tipo AMX. Com isto, a estrutura de 7 foi determinada como 6"- $O$-p-cumaroil- $\beta$-glicopiranosil-3'-metoxicanferol. ${ }^{14}$

O composto 9, obtido como um sólido resinoso amarelo, apresentou a fórmula molecular $\mathrm{C}_{18} \mathrm{H}_{30} \mathrm{O}_{8}$, deduzida através da combinação dos dados de $\mathrm{RMN}{ }^{1} \mathrm{H}$ e ${ }^{13} \mathrm{C}$ (Tabela 1) e espectrometria de massa de alta resolução $\left(\mathrm{m} / \mathrm{z}, 385,1868,[\mathrm{M}-\mathrm{H}]^{-}\right)$. Exceto pelos sinais adicionais em $\delta_{\mathrm{C}} 102,8-62,9 / \delta_{\mathrm{H}} 4,34-3,63$ correspondentes a uma unidade de glicose, todos os demais deslocamentos químicos para 9 mostraram-se semelhantes ao do composto 3 (Tabela 1). Através da correlação a longa distância entre o sinal em $\delta 4.34(d, J=7,8 \mathrm{~Hz}$, H-1') com o carbono em $\delta 77.4$ (C-9) no espectro HMBC, foi possível determinar com segurança a localização da unidade de glicose. Estes dados conduziram à estrutura de um derivado glicosilado do blumenol A, conhecido como roseosídeo, o qual foi previamente isolado de Tapirira guianensis (Anacardiaceae) ${ }^{11}$ e Macaranga tanarius (Euphorbiaceae). ${ }^{19}$

\section{CONCLUSÃO}

O estudo fitoquímico de C. pedicellatus culminou no isolamento de metabólitos secundários de natureza terpênica e fenólica, comuns em espécies de Croton. No entanto, são relatados pela primeira vez na família Euphorbiaceae dois análogos do tilirosídeo, 6"-O-p-cumaroil$\beta$-galactopiranosilcanferol e 6"-O-p-cumaroil- $\beta$-glicopiranosil-3'metoxicanferol, além da alpinumisoflavona, 3-glicopiranosilquercetina e do ácido 4-hidroxi-3,5-dimetoxibenzoico.

\section{MATERIAL SUPLEMENTAR}

Está disponível em http://quimicanova.sbq.org.br, em arquivo pdf, com acesso livre.

\section{AGRADECIMENTOS}

Às agências brasileiras de fomento à pesquisa CNPq/CAPES/ PRONEX e FUNCAP pelas bolsas de estudo, bolsas de pesquisador e auxílios financeiros concedidos para a realização deste trabalho.

\section{REFERÊNCIAS}

1. Webster, G. L.; Missouri Bot. Gard. 1994, 81, 33.

2. Maciel, M. A. M.; Cortez, J. K. P. C.; Gomes, F. E. S.; Revista Fitos 2006, 2, 54 .

3. Carrenho, R.; Bononi, V. L. R.; Barbosa, L. M.; Hoehnea 1997, 24, 107.

4. Salatino, A.; Salatino, M. L. F.; Negri, G.; J. Braz. Chem. Soc. 2007, 18, 11. 
5. Maciel, M. A. M.; Pinto, A. C.; Kaiser, C. R.; Magn. Resson.Chem. 2003, 41, 278

6. Randau, K. P.; Florêncio, D. C.; Ferreira, C. P.; Xavier, H. S.; Rev. Bras. Farmacogn. 2004, 14, 89.

7. Palmeira Júnior, S. F.; Conserva, L. M.; Barbosa Filho, J. M.; Nat. Prod. Comm. (Online) 2006, 1, 319.

8. Brasil, D. S. B.; Muller, A. H.; Guilhon, G. M. S. P.; Alves, C. N.; Peris, G.; Llusar, R.; Moliner, V.; J. Braz. Chem. Soc. 2010, 21, 731.

9. Park, Y.; Moon, B.; Yang, H.; Lee, Y.; Lee, E.; Lim, Y.; Magn. Resson. Chem. 2007, 45, 1072; Vega, M. R. G.; Esteves-Souza, A.; Vieira, I. J. C.; Mathias, L.; Braz-Filho, B.; Echevarria, A.; J. Braz. Chem. Soc. 2007, 18, 1554

10. El-Masry, S.; Amer, M. E.; Abdel-Kader, M. S.; Zaatout, H. H.; Phytochemistry 2002, 60, 783.

11. Gonzalez, A. G.; Guillermo, J. A.; Ravelo, A. G.; Jimenez, I. A.; J. Nat. Prod. 1994, 57, 400; Bu, W.; Shi, Y.; Yan, Y.; Lu, Q.; Liu, G.; Li, Y.; Cheng, Y.; Nat. Prod. Bioprospect. 2011, 1, 134.

12. Kanchanapoom, T.; Phytochemistry, 2007, 68, 692.

13. Silva, D. A.; Costa, D. A.; Silva, D. F.; Souza, M. F. V.; Agra, M. F.; Medeiros, I. A.; Barbosa-Filho, J. M.; Braz-Filho, R.; Rev. Bras. Farmacogn. 2005, 15, 1 .
14. Gobbo-Neto, L.; Lopes, N. P.; J. Agric. Food Chem. 2008, 56, 1193.

15. Stark, T.; Bareuther, S.; Hofmann, T.; J. Agric. Food Chem. 2005, 53, 5407.

16. Correia, S. J.; David, J. M.; Silva, E. P.; David, J. P.; Lopes, L. M. X.; Guedes, M. L. S.; Quim. Nova 2008, 31, 2056.

17. Erosa-Rejón, G.; Peña-Rodríguez, L. M.; Sterner, O.; J. Mex. Chem. Soc. 2009, 53, 44; Kato-Noguchi, H.; Tamura, K.; Sasaki, H.; Suenaga, K.; J. Plant Physiol. 2012, 169, 682.

18. Aderogba, M. A.; McGaw, L. J.; Bezabih, M.; Abegaz, B. M.; Nat. Prod. Res. 2011, 25, 1224; Zou, G.; Su, Z.; Zhang, H.; Wang, Y.; Yang, J.; Zou, Z.; Molecules 2010, 15, 1097; Palmeira, S. F. J.; Alves, V. L.; Moura, F. S.; Vieira, L. F. A.; Conserva, L. M.; Lemos, R. P. L.; Rev. Bras. Farmacogn. 2006, 16, 397; Phan, M.; Lee, J.; Phan, T.; Tap Chi Hoa Hoc. 2004, 42, 125; Lencina, C.; Pires, V. S.; Gosmann, G.; Taketa, A. T. C.; Schenkel, E. P.; Rev. Bras. Farmacogn. 2001, 11, 89.

19. Matsunami, K.; Otsuka, H.; Kondo, K.; Shinzato, T.; Kawahata, M.;Yamaguchi, K.; Takeda, Y.; Phytochemistry 2009, 70, 1277. 


\section{FLAVONOIDES E SESQUITERPENOS DE Croton pedicellatus Kunth.\#}

Elton Luz Lopes, Manoel Andrade Neto, Edilberto Rocha Silveira e Otilia Deusdênia Loiola Pessoa*

Departamento de Química Orgânica e Inorgânica, Universidade Federal do Ceará, CP 12200, 60971-270 Fortaleza - CE, Brasil Raimundo Braz Filho ${ }^{\&}$

Departamento de Química, Universidade Estadual do Norte Fluminense, 28013-602 Campos dos Goytacazes - RJ, Brasil

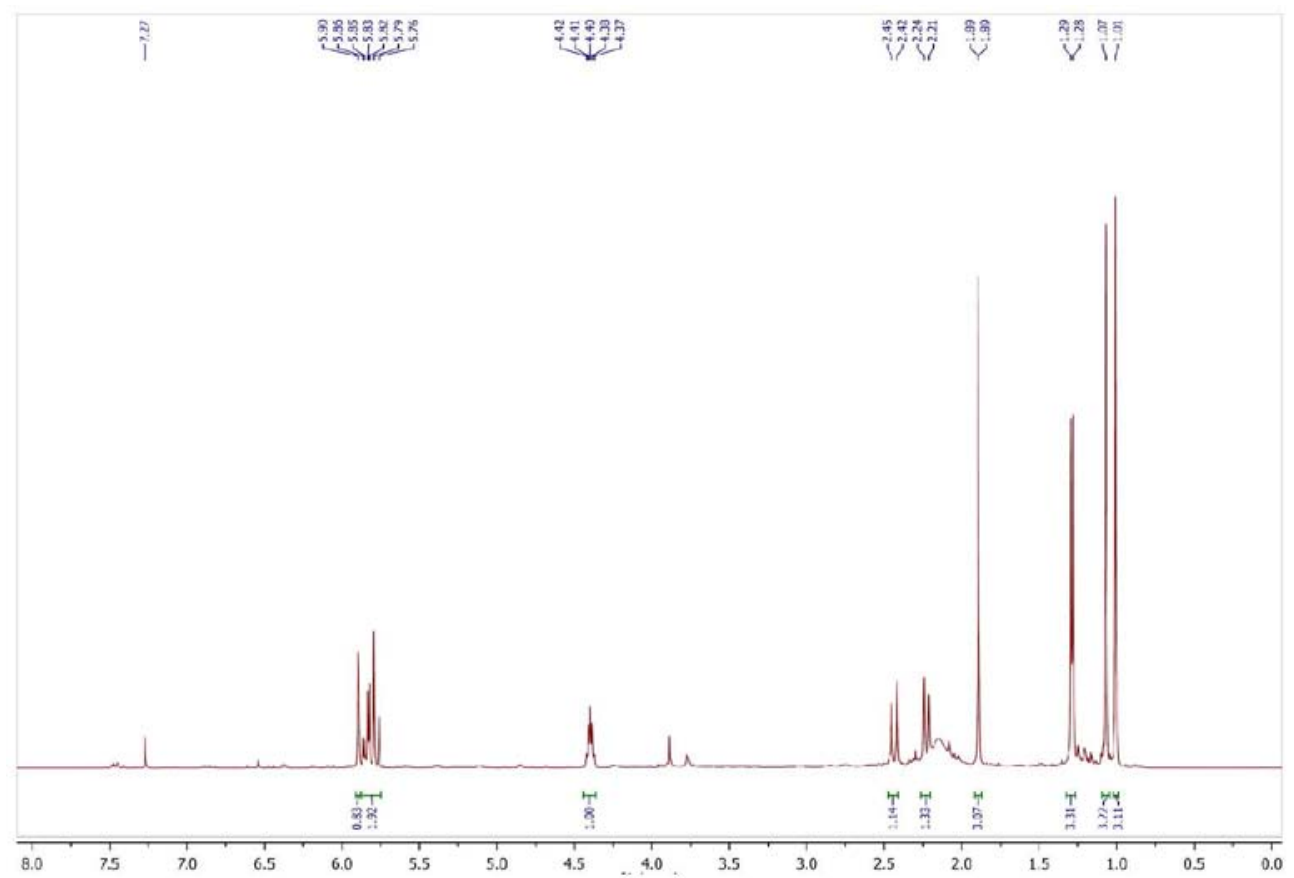

Figura 1S. Espectro de $\mathrm{RMN}^{l} \mathrm{H}\left(500 \mathrm{MHz}, \mathrm{CDCl}_{3}\right)$ de 3

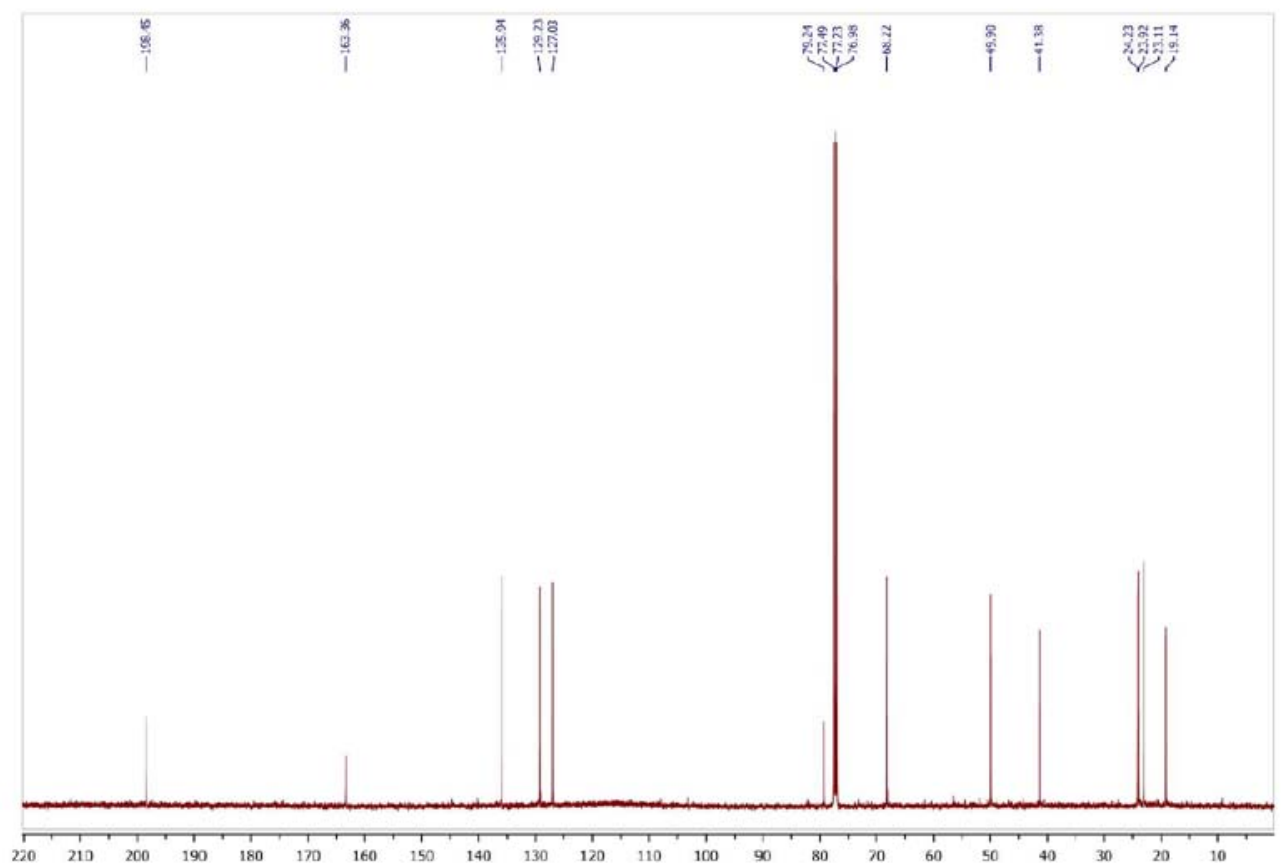

Figura 2S. Espectro de $\mathrm{RMN}^{13} \mathrm{C}\left(125 \mathrm{MHz}, \mathrm{CDCl}_{3}\right)$ de 3 


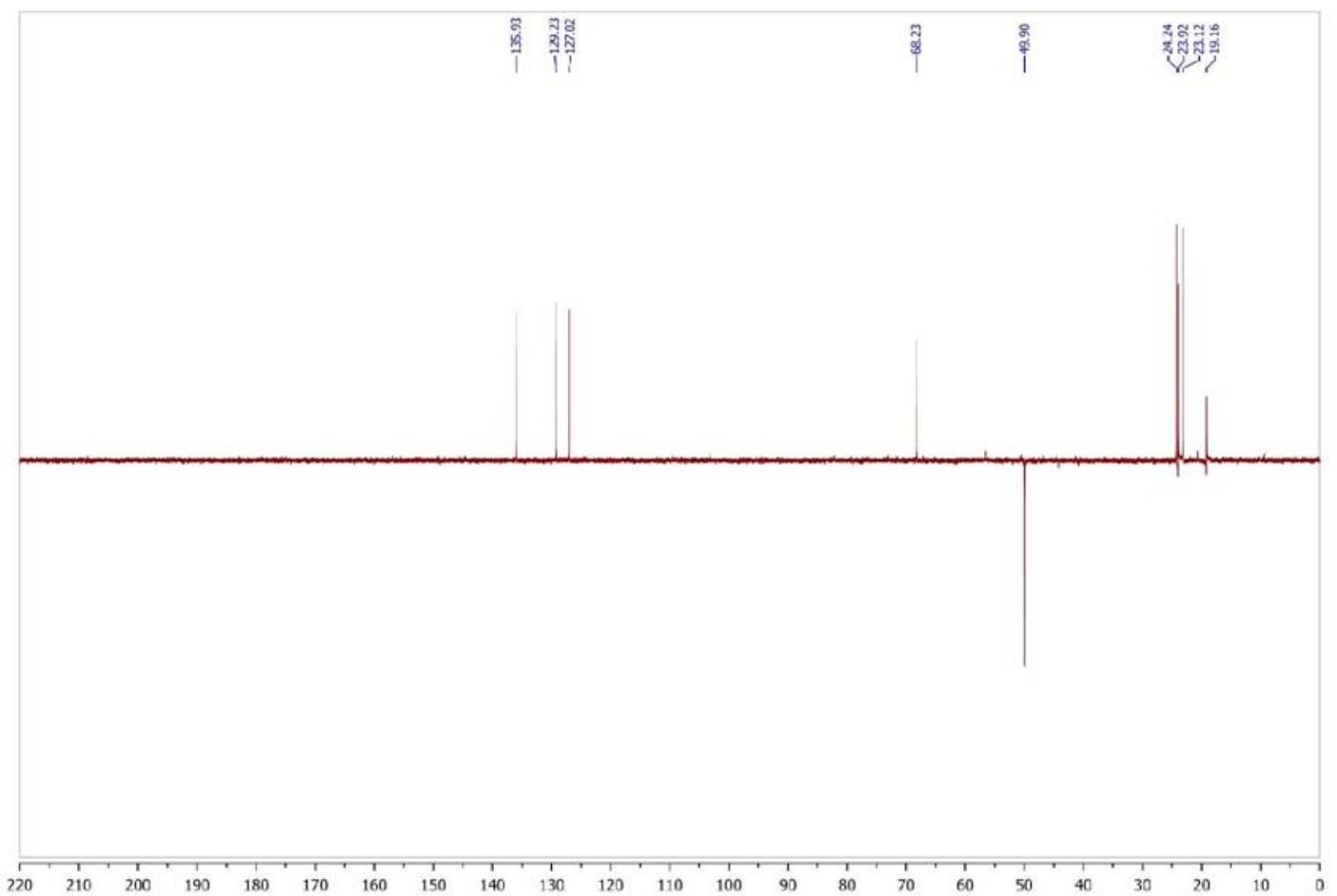

Figura 3S. Espectro de $\mathrm{RMN}^{13} \mathrm{C}-\mathrm{DEPT} 135\left(125 \mathrm{MHz}, \mathrm{CDCl}_{3}\right)$ de 3

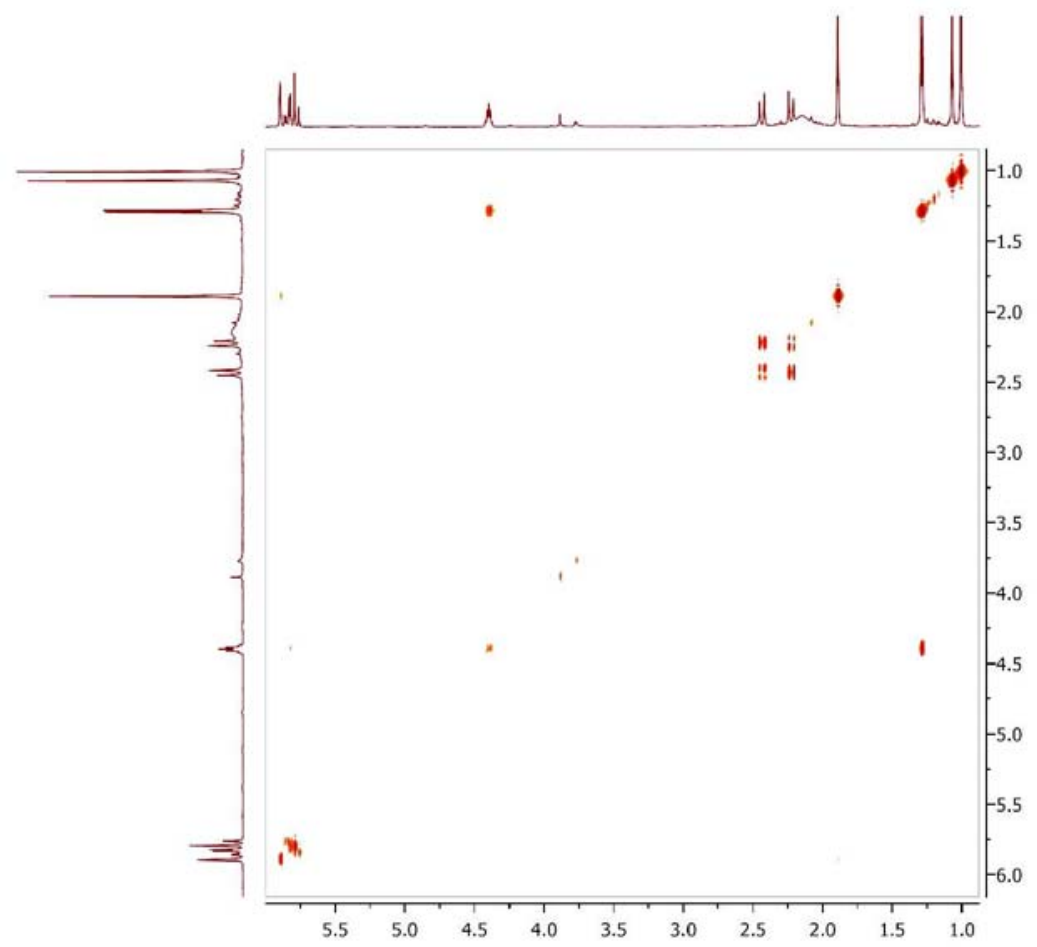

Figura 4S. Espectro de $\operatorname{COSY}\left(500 \mathrm{MHz}_{2} \mathrm{CDCl}_{3}\right)$ de 3 


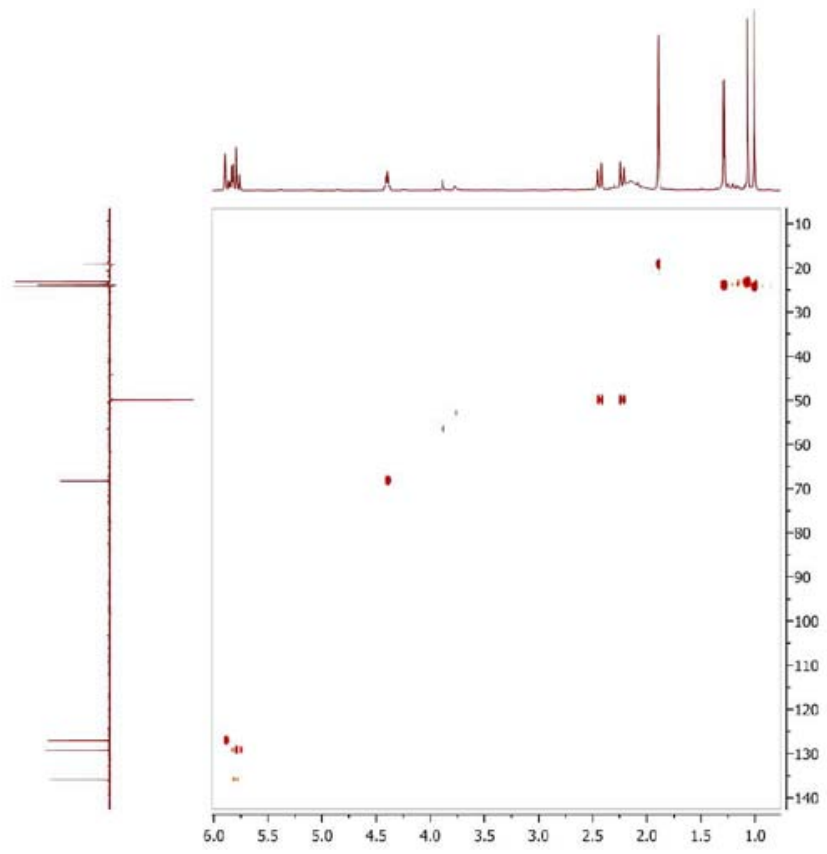

Figura 5S. Espectro de $\mathrm{HSQC}\left(500 / 125 \mathrm{MHz}, \mathrm{CDCl}_{3}\right)$ de 3

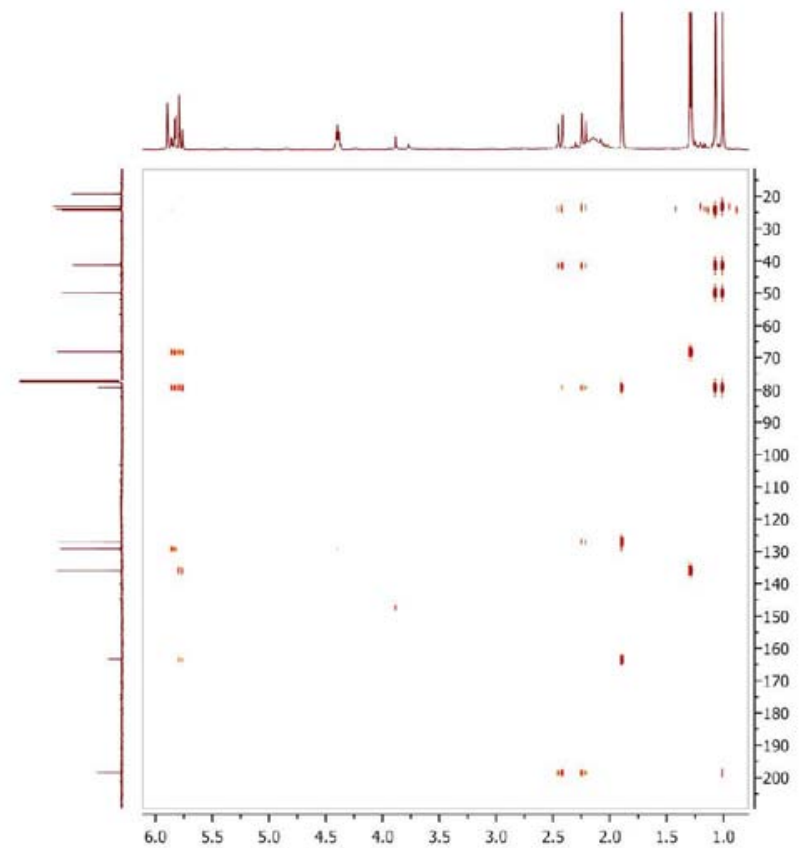

Figura 6S. Espectro de $\mathrm{HMBC}\left(500 / 125 \mathrm{MHz}, \mathrm{CDCl}_{3}\right)$ de 3 


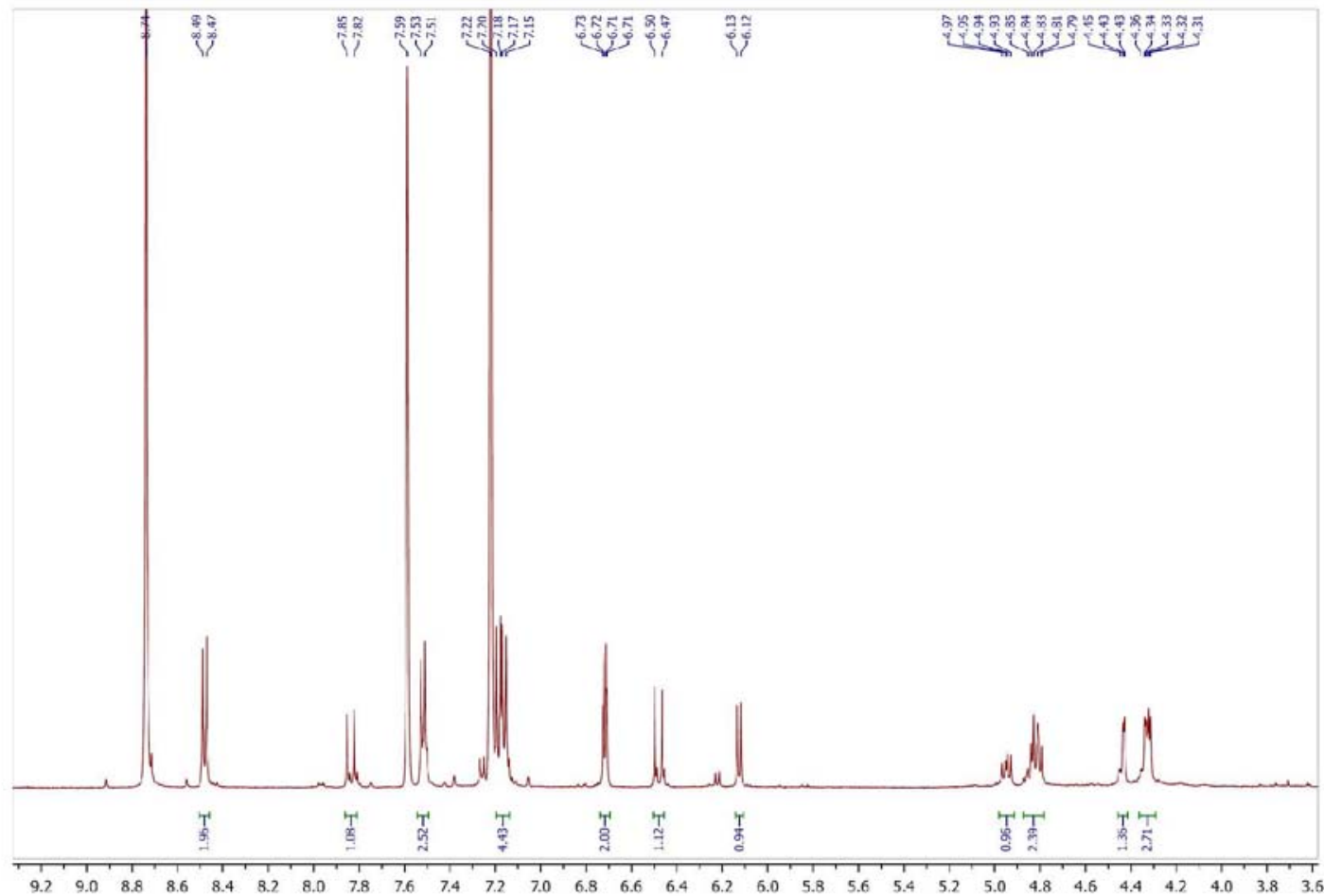

Figura 7S. Espectro de $R M N^{1} H\left(500 \mathrm{MHz}, C_{5} D_{5} N\right)$ de 5

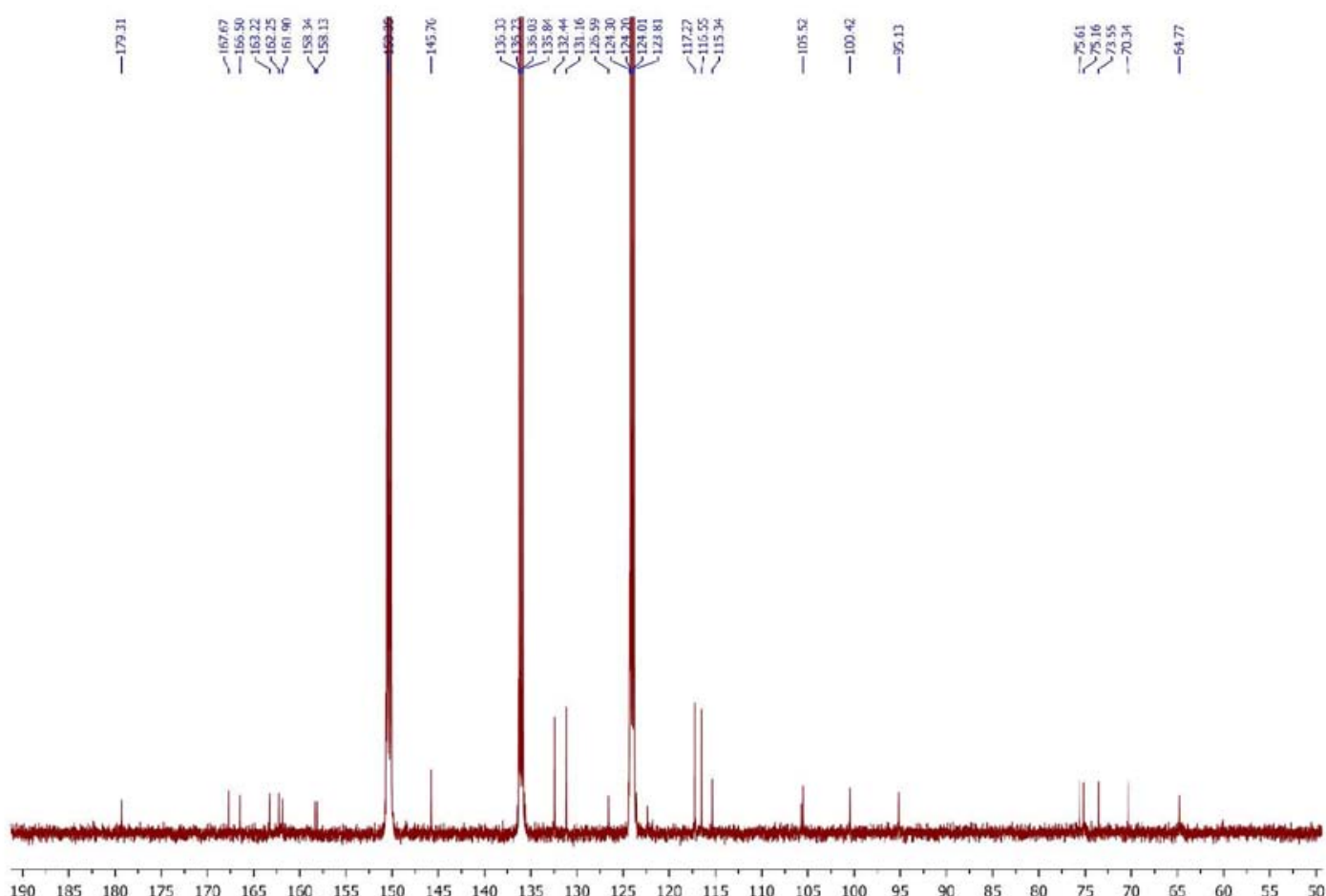

Figura 8S. Espectro de $R M N{ }^{13} \mathrm{C}\left(125 \mathrm{MHz}, \mathrm{C}_{5} \mathrm{D}_{5} \mathrm{~N}\right)$ de 5 


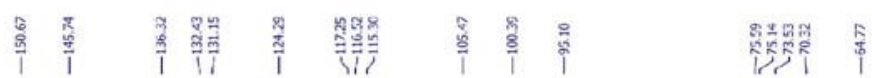

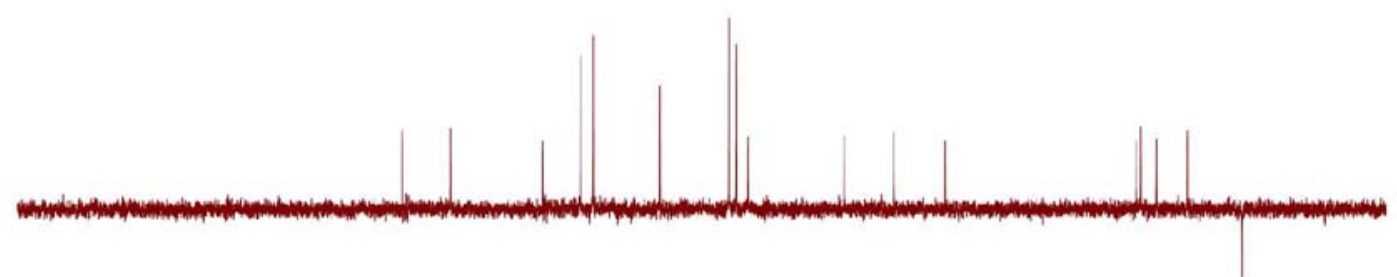

$\begin{array}{lllllllllllllllllllllllllllllllllll}190 & 185 & 180 & 175 & 170 & 165 & 160 & 155 & 150 & 145 & 140 & 135 & 130 & 125 & 120 & 115 & 110 & 105 & 100 & 95 & 90 & 85 & 30 & 75 & 70 & 65 & 60 & 55 & 50\end{array}$

Figura 9S. Espectro de $R M N{ }^{13} \mathrm{C}-\mathrm{DEPT} 135\left(125 \mathrm{MHz}, \mathrm{C}_{5} \mathrm{D}_{5} \mathrm{~N}\right)$ de 5

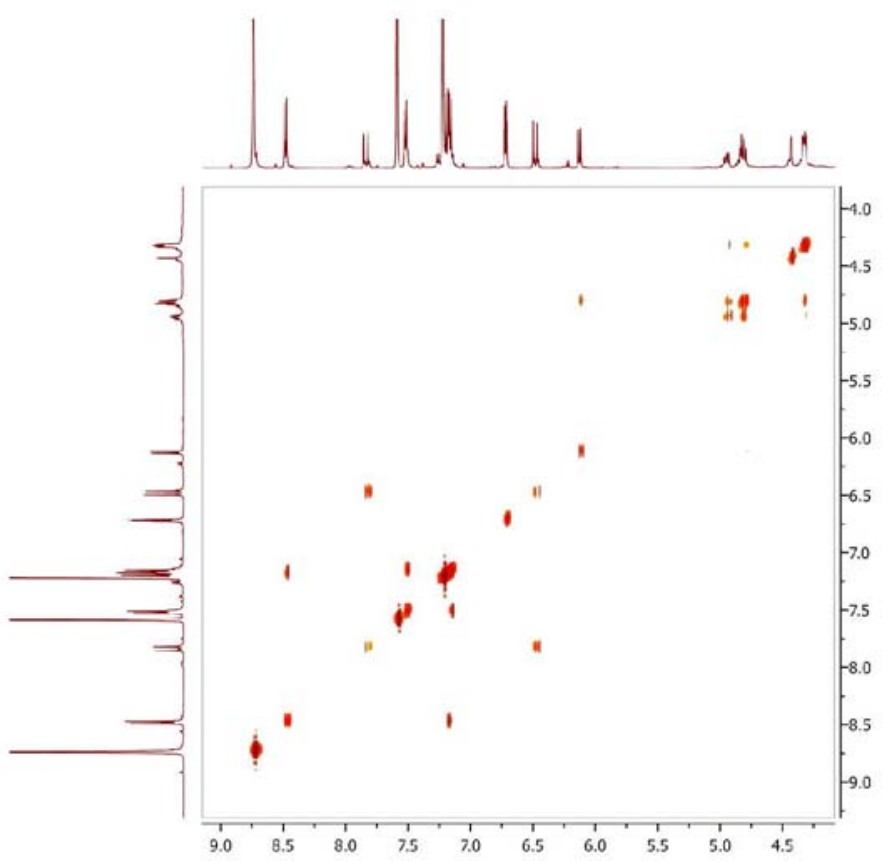

Figura 10S. Espectro de $\operatorname{COSY}\left(500 \mathrm{MHz}, \mathrm{C}_{5} \mathrm{D}_{5} \mathrm{~N}\right)$ de 5 


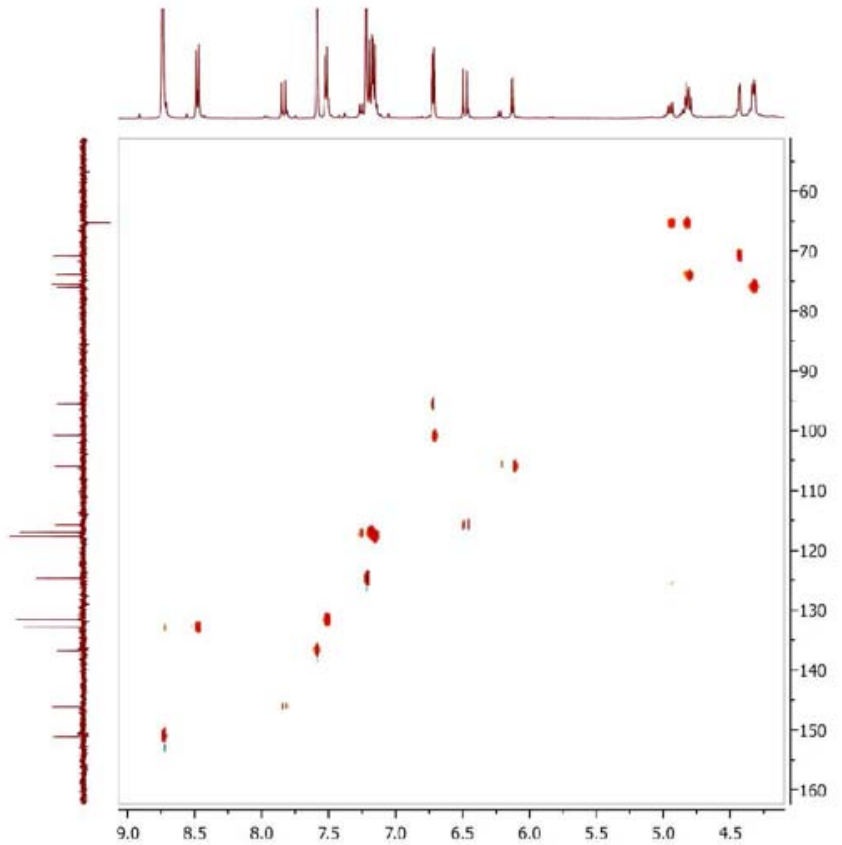

Figura 11S. Espectro de HSQC $\left(500 / 125 \mathrm{MHz}, \mathrm{C}_{5} D_{5} \mathrm{~N}\right)$ de 5

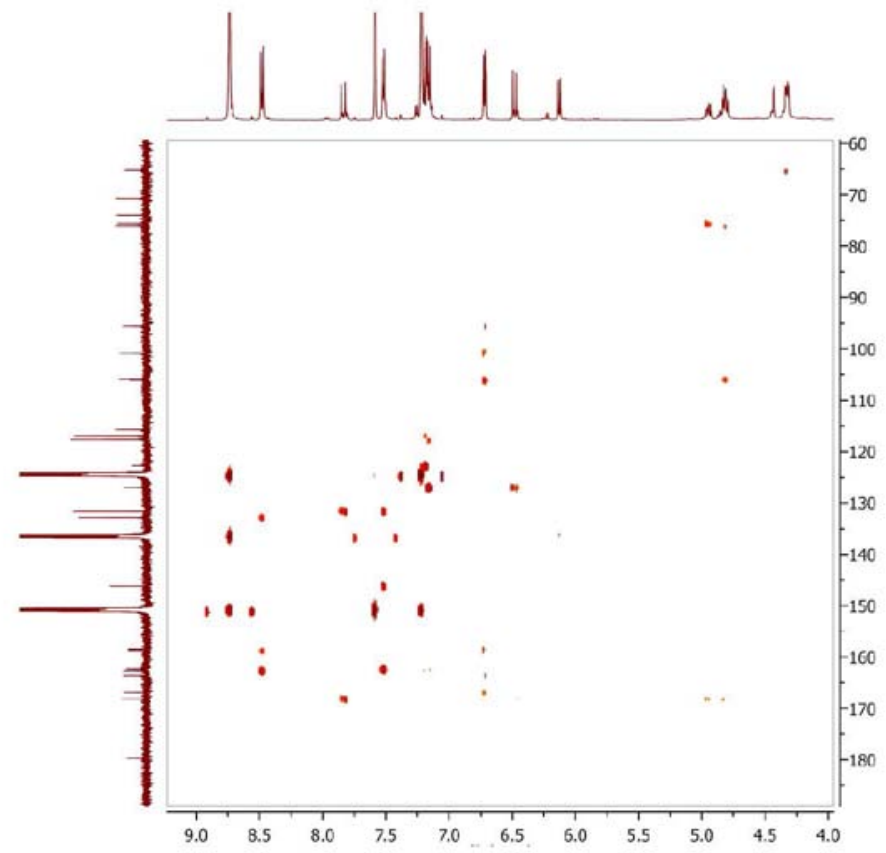

Figura 12S. Espectro de $\mathrm{HMBC}\left(500 / 125 \mathrm{MHz}, \mathrm{C}_{5} \mathrm{D}_{5} \mathrm{~N}\right)$ de 5 


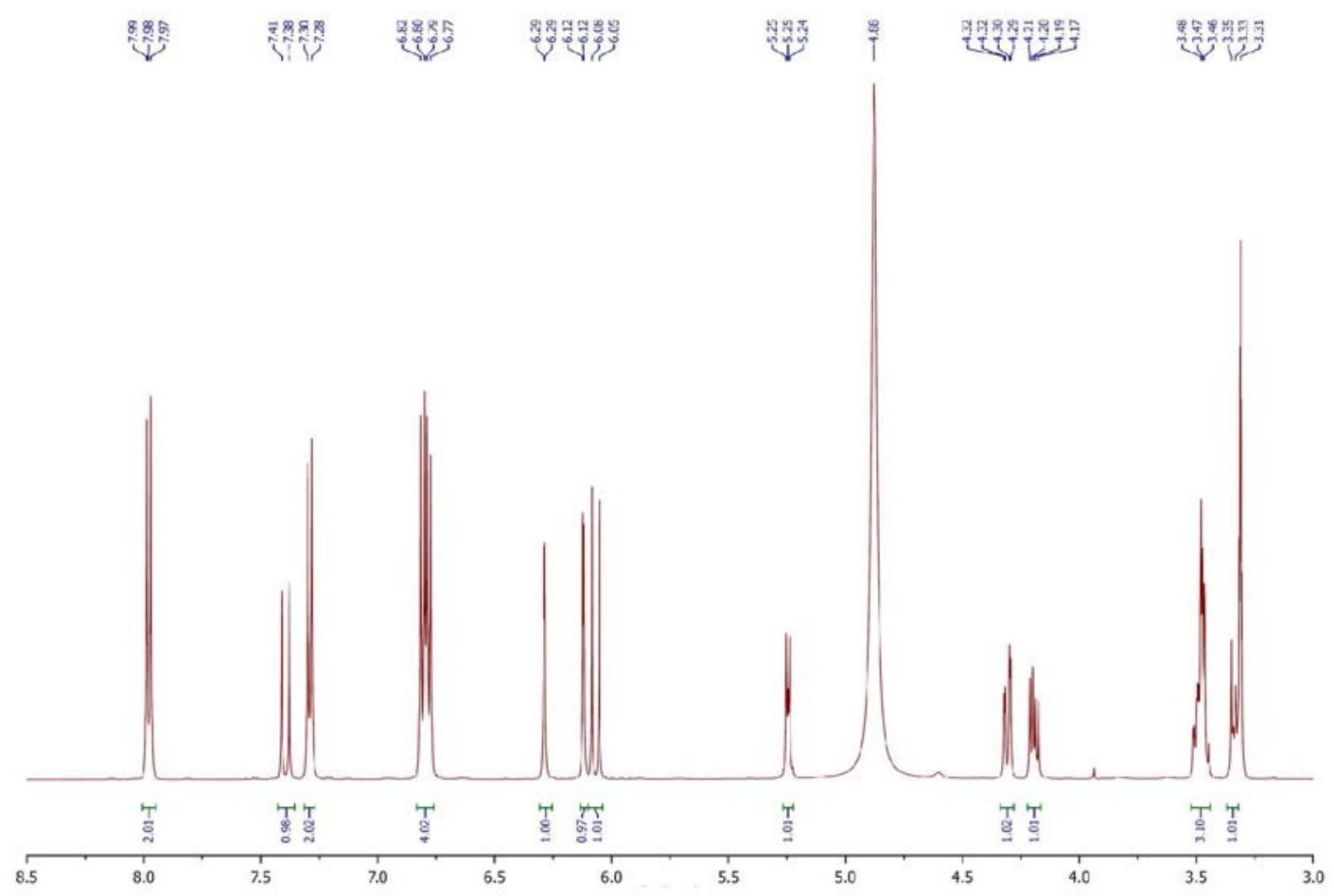

Figura 13S. Espectro de $\mathrm{RMN}^{1} \mathrm{H}\left(500 \mathrm{MHz}, \mathrm{CD}_{3} \mathrm{OD}\right)$ de 6
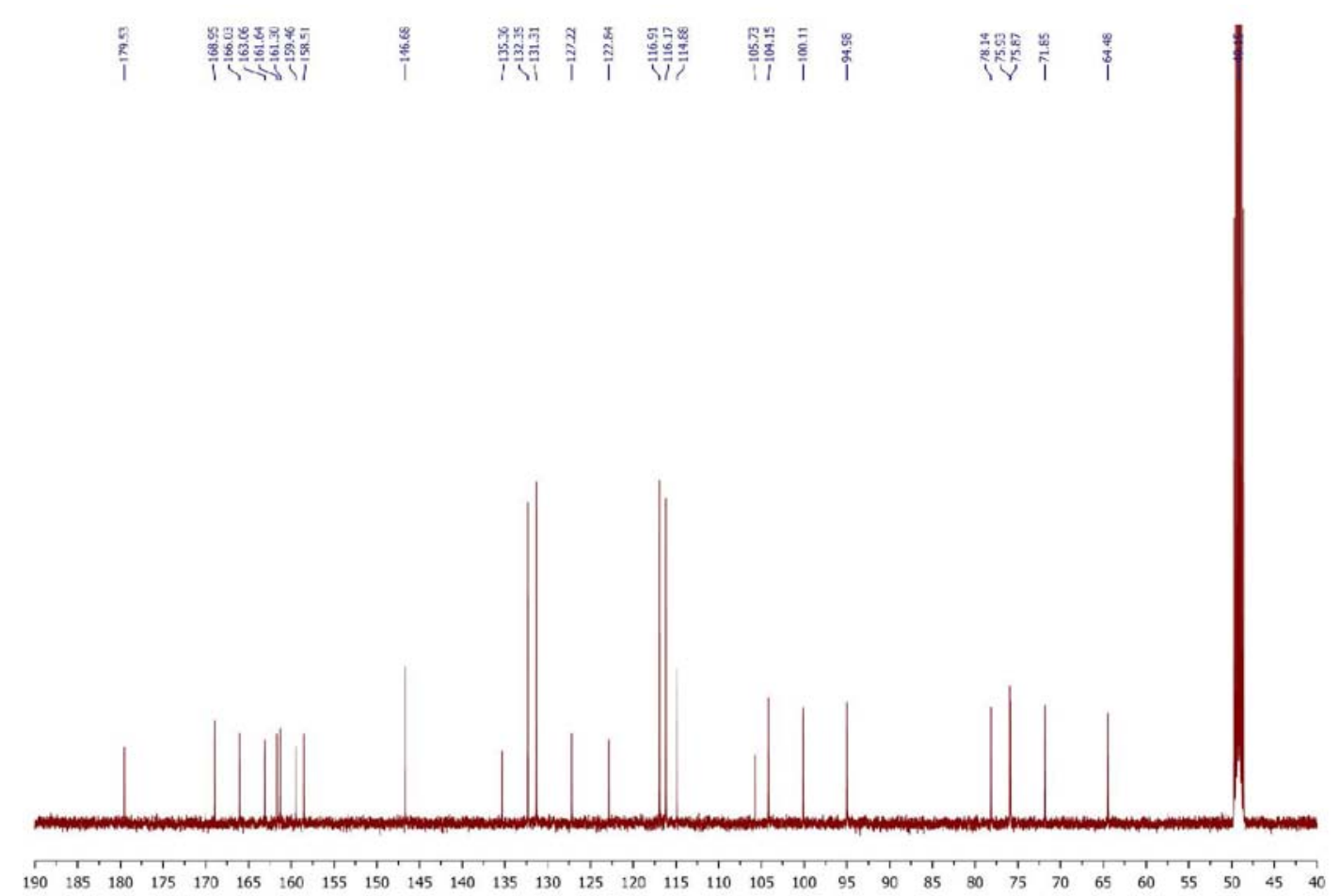

Figura 14S. Espectro de $\mathrm{RMN}^{13} \mathrm{C}\left(125 \mathrm{MHz}, \mathrm{CD}_{3} \mathrm{OD}\right)$ de 6 


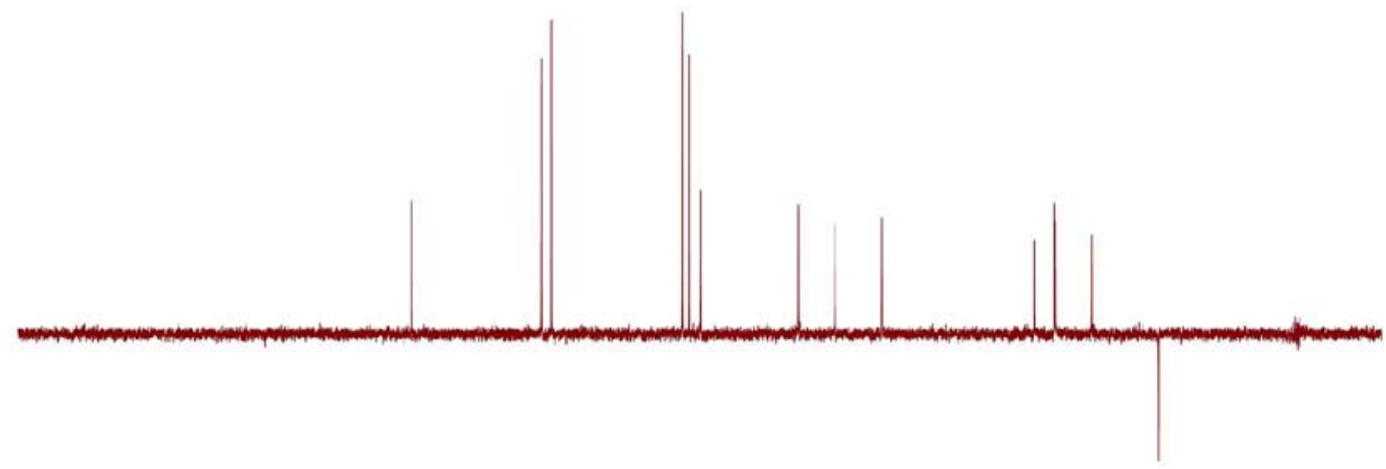

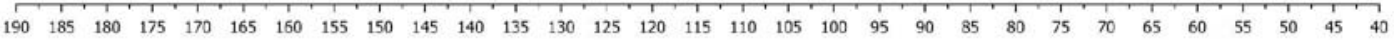

Figura 15S. Espectro de $R M N{ }^{13} \mathrm{C}$-DEPT $135\left(125 \mathrm{MHz}, C D_{3} \mathrm{OD}\right)$ de 6

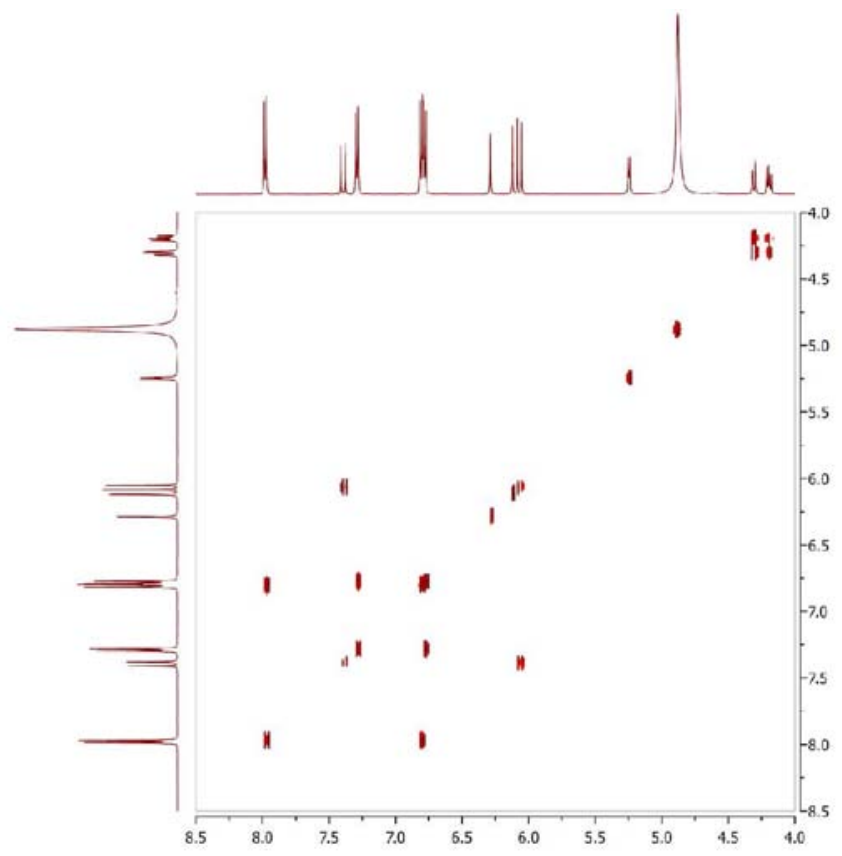

Figura 16S. Espectro de $\operatorname{COSY}\left(500 \mathrm{MHz}, \mathrm{CD}_{3} \mathrm{OD}\right)$ de 6 


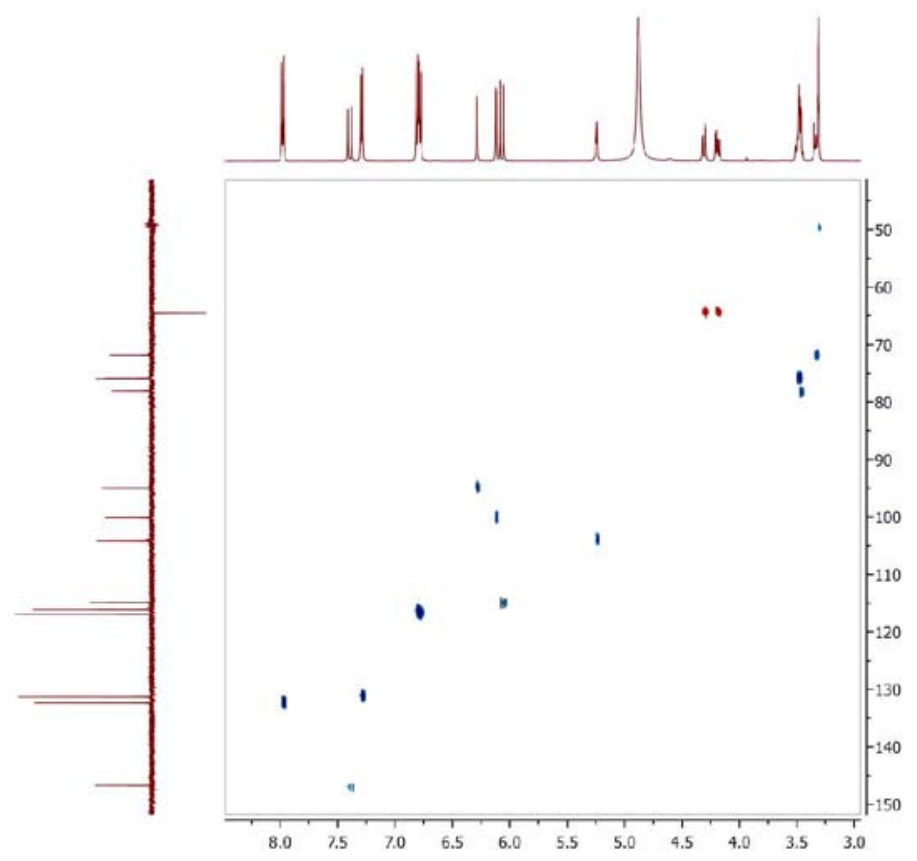

Figura 17S. Espectro de $\operatorname{HSQC}\left(500 / 125 \mathrm{MHz}, C D_{3} \mathrm{OD}\right)$ de 6

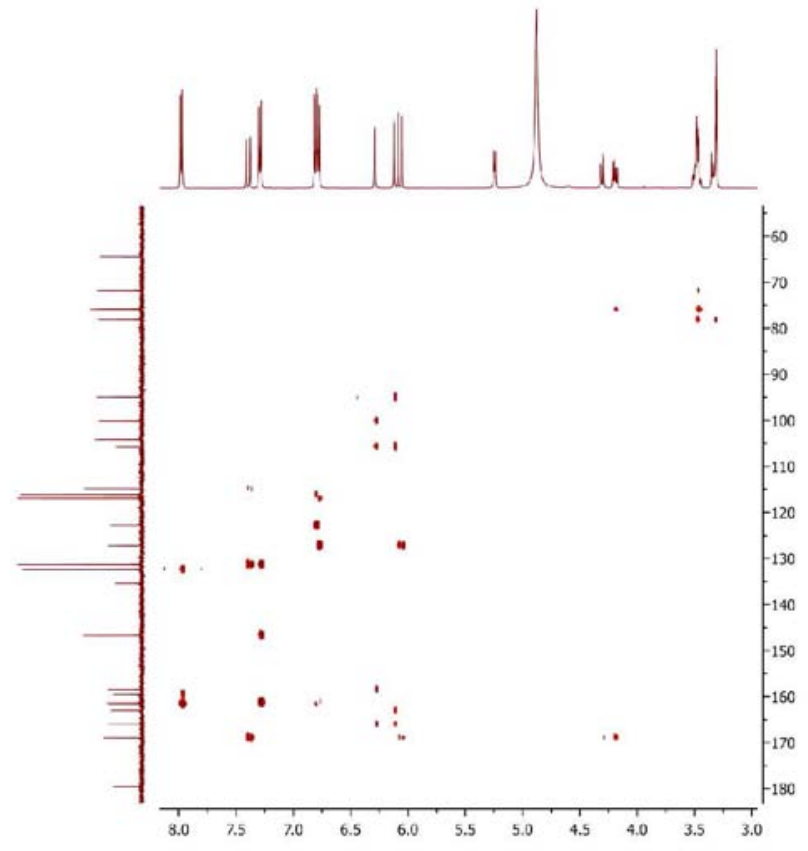

Figura 18S. Espectro de $\mathrm{HMBC}\left(500 / 125 \mathrm{MHz}, \mathrm{CD}_{3} \mathrm{OD}\right)$ de 6 


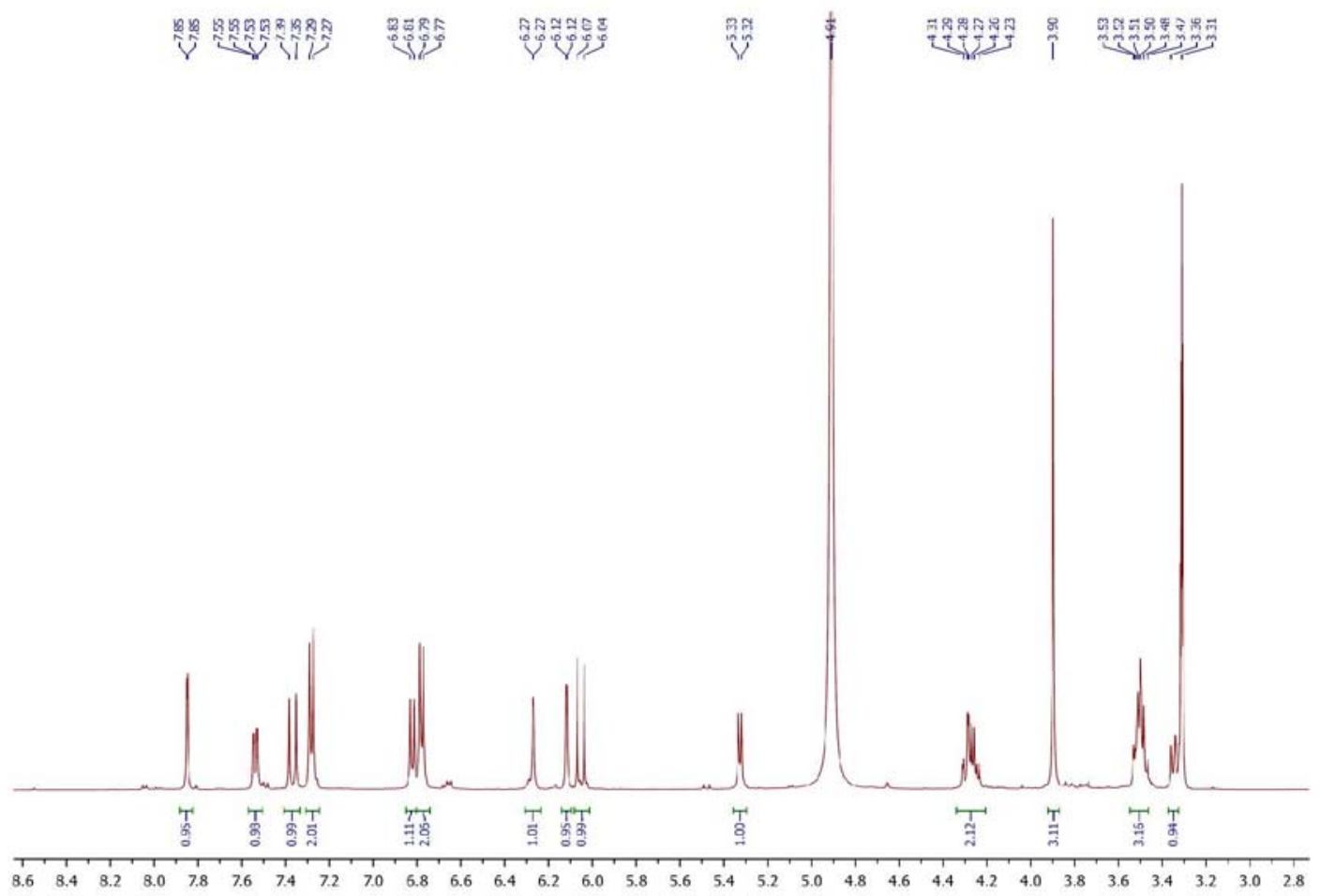

Figura 19S. Espectro de $\mathrm{RMN}^{l} \mathrm{H}\left(500 \mathrm{MHz}, \mathrm{CD}_{3} \mathrm{OD}\right)$ de 7

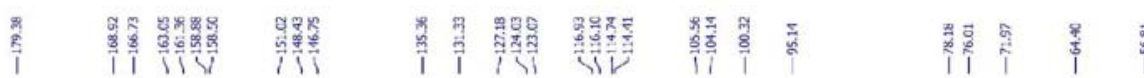

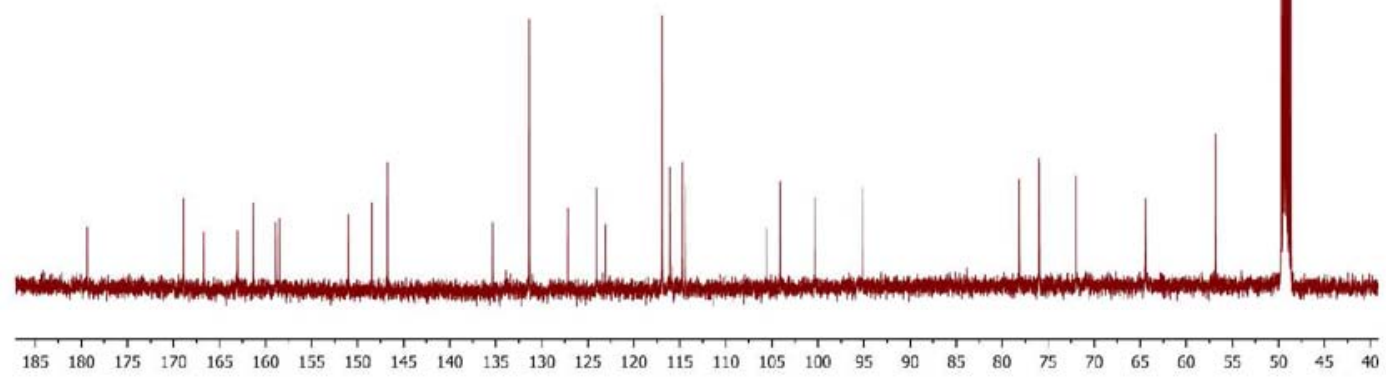

Figura 20S. Espectro de $R M N^{13} \mathrm{C}\left(125 \mathrm{MHz}, C D_{3} \mathrm{OD}\right)$ de 7 


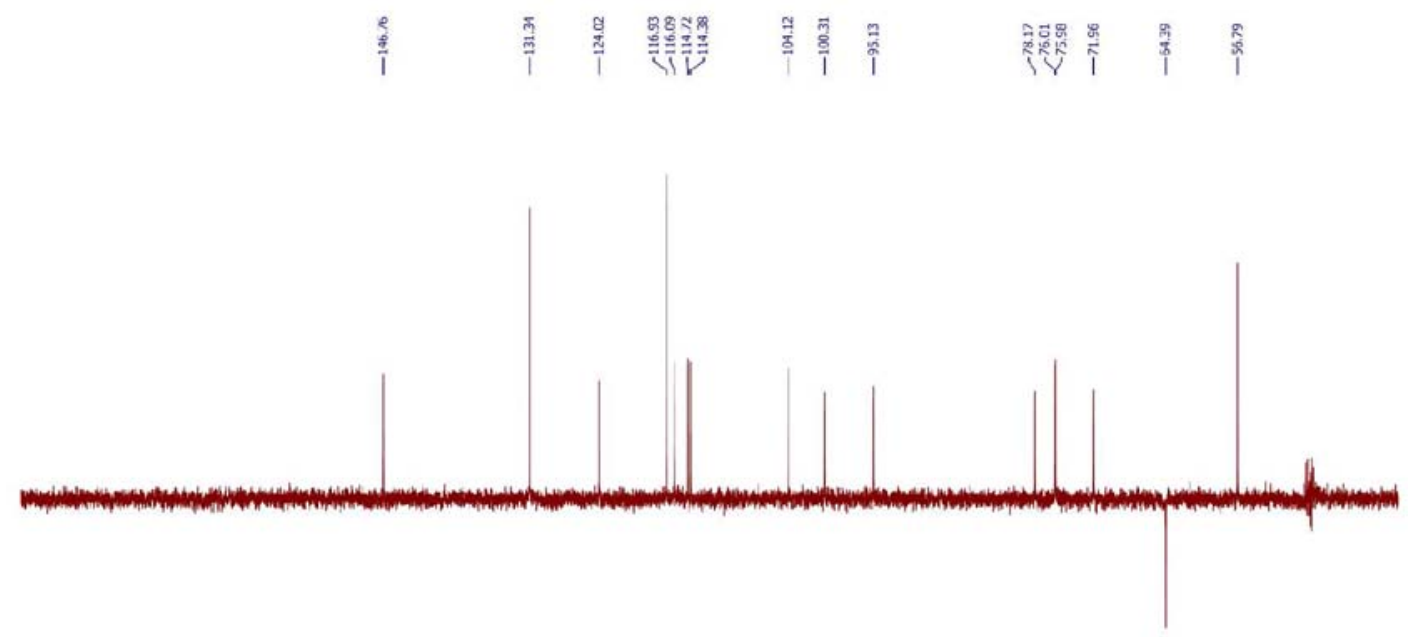

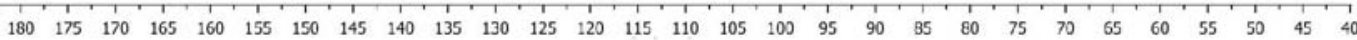

Figura 21S. Espectro de $R M N{ }^{13} \mathrm{C}-\mathrm{DEPT} 135\left(125 \mathrm{MHz}, \mathrm{CD}_{3} \mathrm{OD}\right)$ de 7

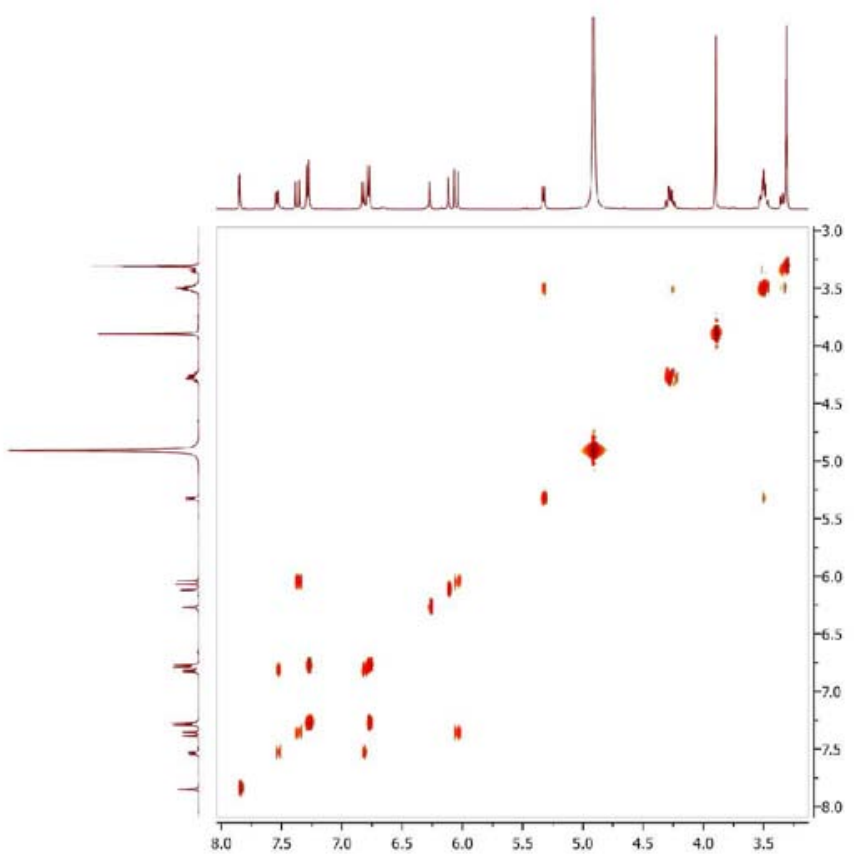

Figura 22S. Espectro de $\operatorname{COSY}\left(500 \mathrm{MHz}, \mathrm{CD}_{3} \mathrm{OD}\right)$ de 7 


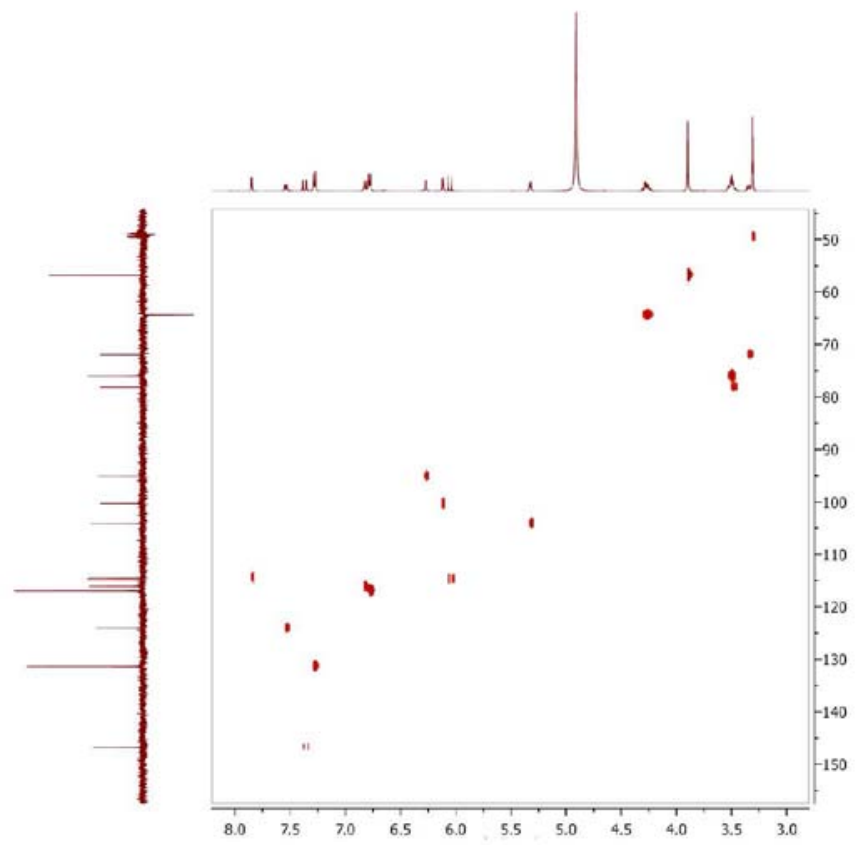

Figura 23S. Espectro de $\mathrm{HSQC}\left(500 / 125 \mathrm{MHz}, \mathrm{CD}_{3} \mathrm{OD}\right)$ de 7

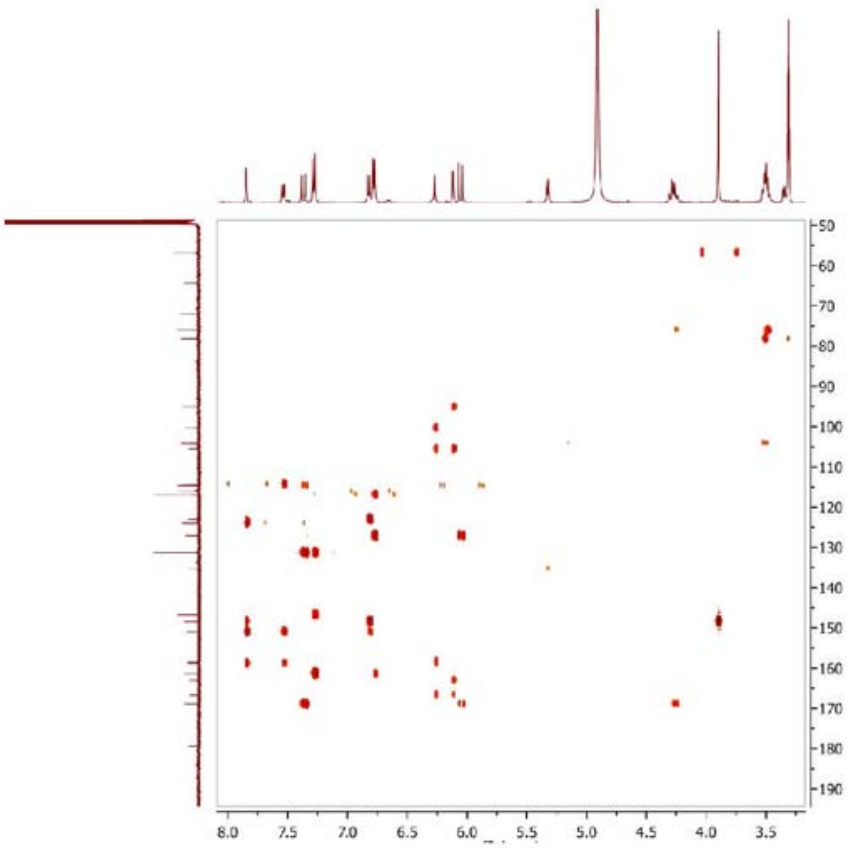

Figura 24S. Espectro de $\mathrm{HMBC}\left(500 / 125 \mathrm{MHz}, C D_{3} \mathrm{OD}\right)$ de 7 


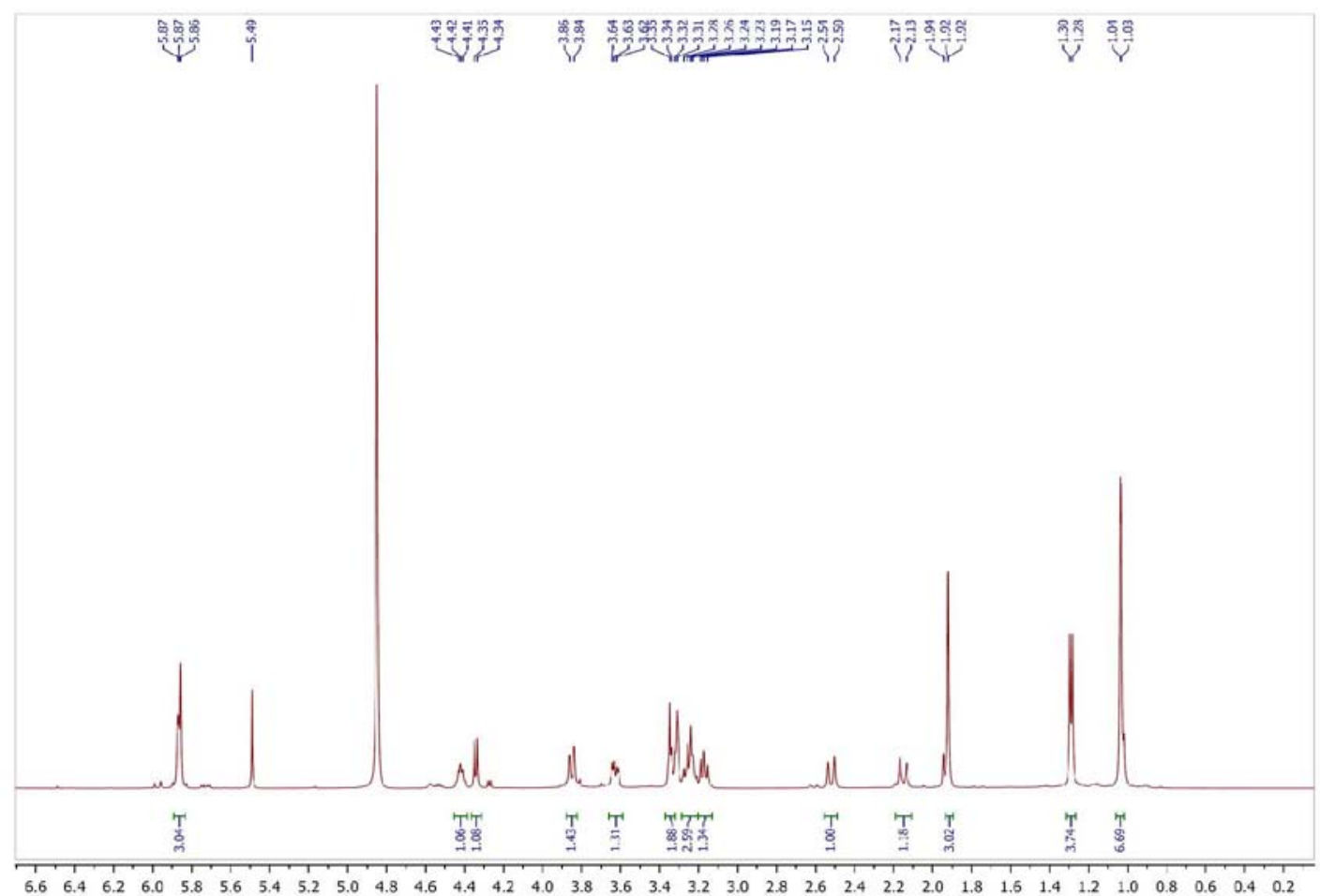

Figura 25S. Espectro de $\mathrm{RMN}^{1} \mathrm{H}\left(500 \mathrm{MHz}, \mathrm{CD}_{3} \mathrm{OD}\right) \mathrm{de} \boldsymbol{9}$

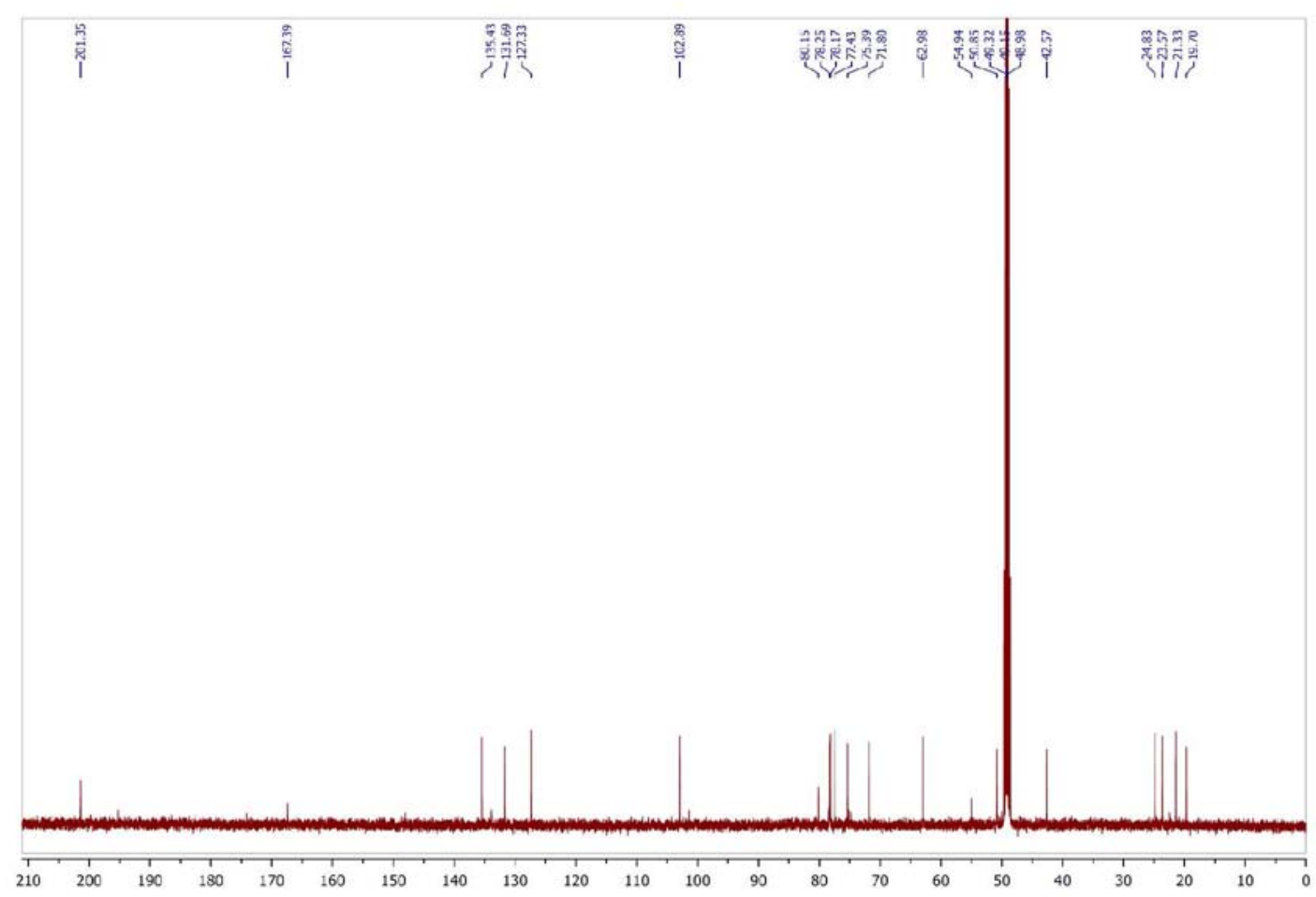

Figura 26S. Espectro de $\mathrm{RMN}^{13} \mathrm{C}\left(125 \mathrm{MHz}, \mathrm{CD}_{3} \mathrm{OD}\right)$ de 9 
$\mathrm{S} 14$

Lopes et al.

Quim. Nova

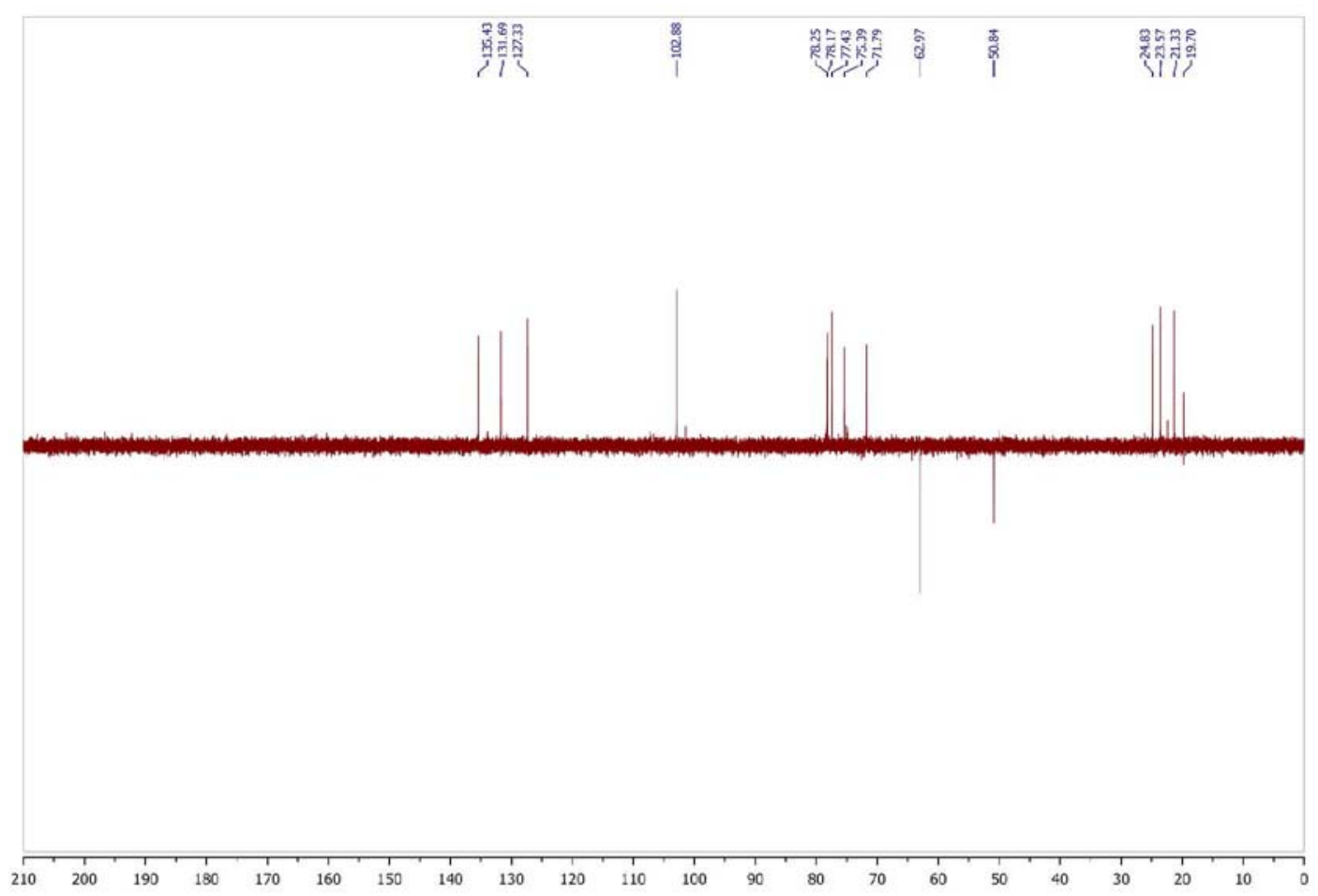

Figura 27S. Espectro de $R M N{ }^{13} \mathrm{C}$-DEPT $135\left(125 \mathrm{MHz}, C D_{3} \mathrm{OD}\right)$ de 9

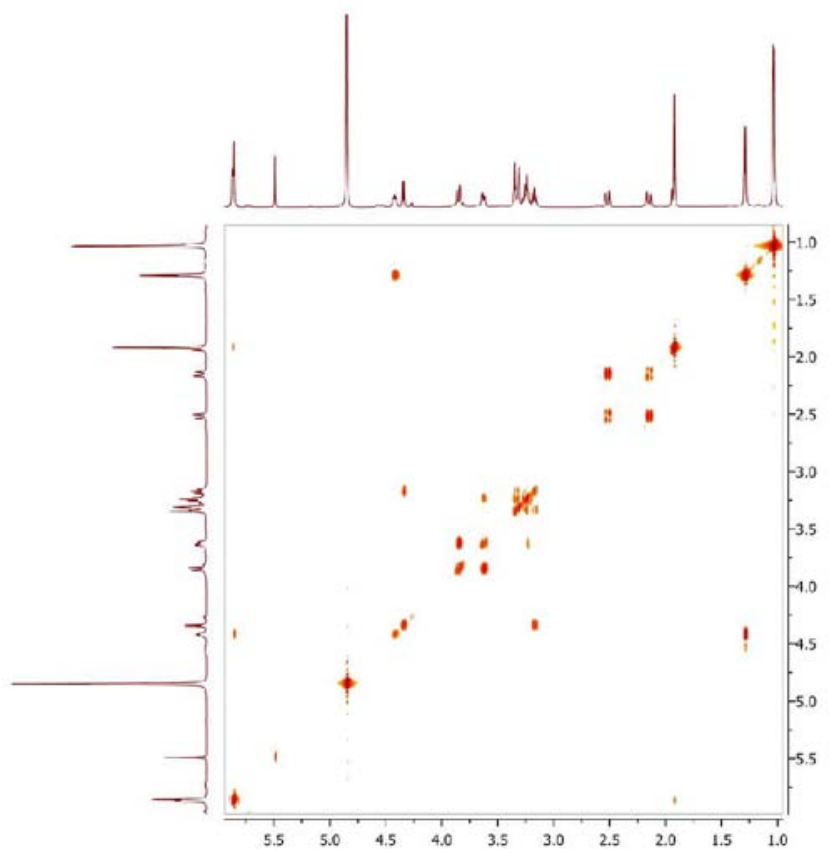

Figura 28S. Espectro de $\mathrm{COSY}\left(500 \mathrm{MHz}, \mathrm{CD}_{3} \mathrm{OD}\right)$ de 9 


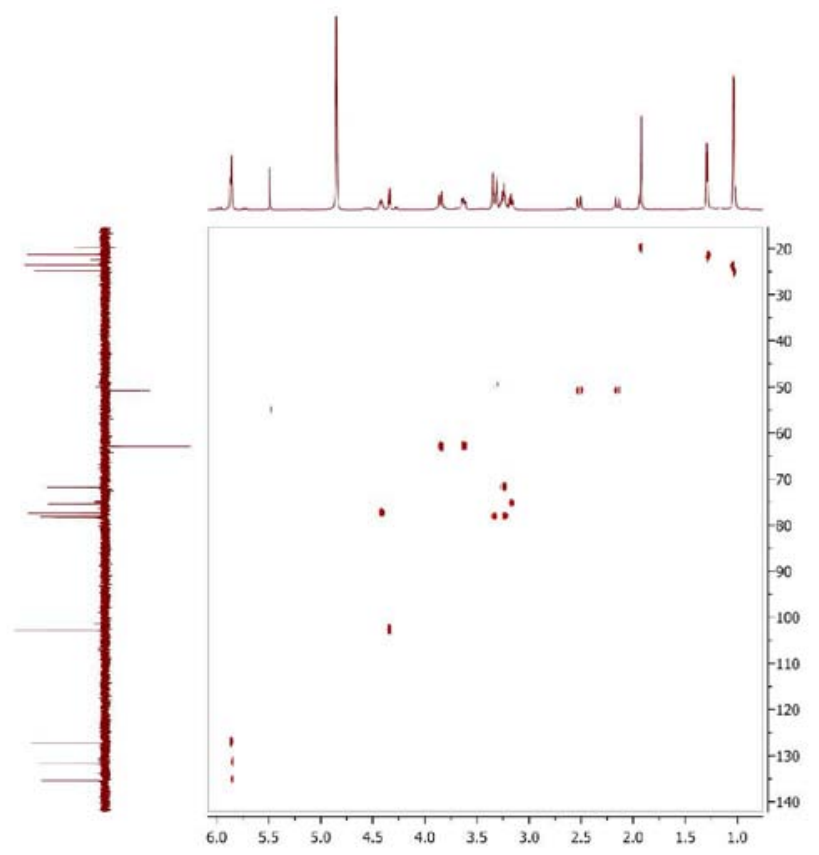

Figura 29S. Espectro de $\operatorname{HSQC}\left(500 / 125 \mathrm{MHz}, C D_{3} \mathrm{OD}\right)$ de 9

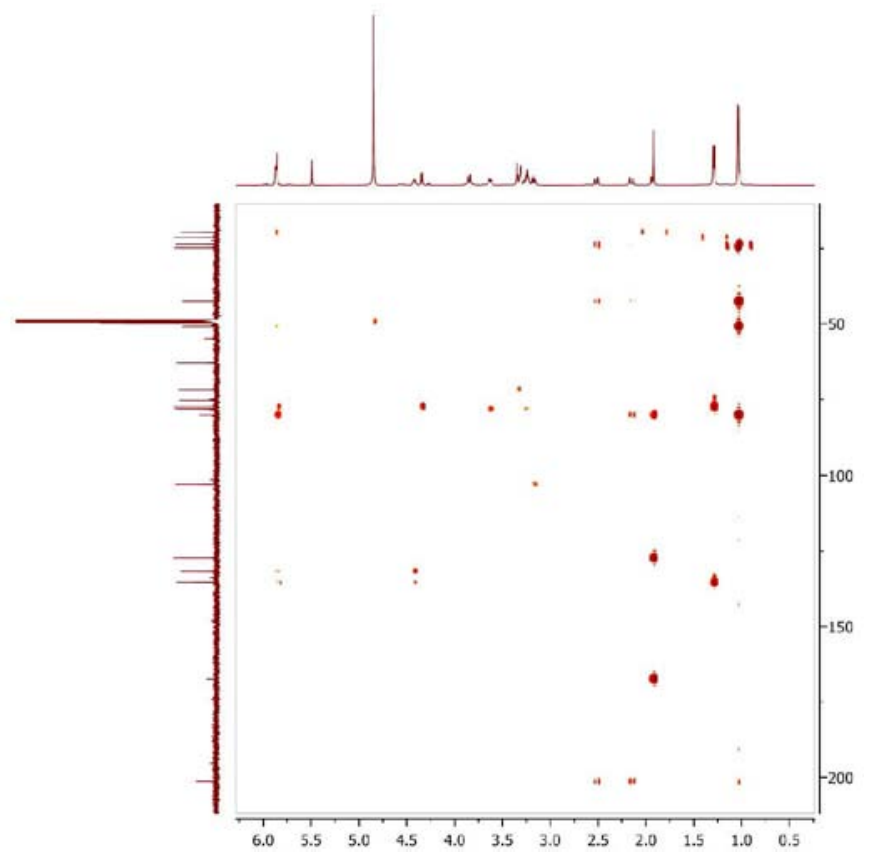

Figura 30S. Espectro de $\mathrm{HMBC}\left(500 / 125 \mathrm{MHz}, \mathrm{CD}_{3} \mathrm{OD}\right)$ de 9 